\title{
Plane-parallel waves as duals of the flat background III: T-duality with torsionless $B$-field
}

\author{
Ladislav Hlavatý* \\ Faculty of Nuclear Sciences and Physical Engineering, \\ Ivo Petr ${ }^{\dagger}$ \\ Faculty of Information Technology, \\ Filip Petrásek ${ }^{\ddagger}$ \\ Faculty of Nuclear Sciences and Physical Engineering \\ Czech Technical University in Prague
}

August 13, 2018

\begin{abstract}
By addition of non-zero, but torsionless $B$-field, we expand the classification of (non-)Abelian T-duals of the flat background in four dimensions with respect to one-, two-, three-, and four-dimensional subgroups of Poincaré group. We discuss the influence of the additional $B$-field on the process of dualization and identify essential parts of the torsionless $B$-field that cannot be eliminated in general by coordinate or gauge transformation of the dual background. These effects are demonstrated using particular examples. Due to their physical importance, we focus on duals whose metrics represent plane-parallel waves. Besides the previously found metrics, we find new pp-waves depending on parameters originating from the torsionless $B$-field. These pp-waves are brought into their standard forms in Brinkmann and Rosen coordinates.
\end{abstract}

\section{Contents}

1 Introduction 2

2 Theoretical background 3

2.1 Dualization with $B$-field . . . . . . . . . . . . . . . . 3

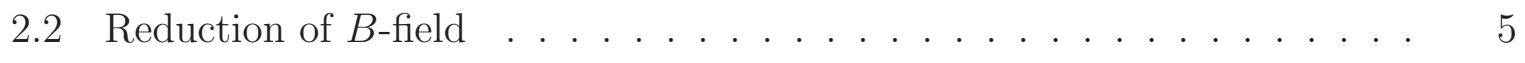

2.3 Plane-parallel waves . . . . . . . . . . . . . . . . . . . . . . 7

3 Duals with respect to one-dimensional subgroups 9

*ladislav.hlavaty@fjfi.cvut.cz

†ivo.petr@fit.cvut.cz

${ }_{\ddagger}^{\ddagger}$ filip.petrasek@fjfi.cvut.cz 
4 Duals with respect to two-dimensional subgroups

4.1 Dualization with respect to subgroup generated by $S_{2,2} \ldots \ldots$. . . . . 10

4.2 Dualization with respect to subgroup generated by $S_{2,3} \ldots \ldots \ldots \ldots$

4.3 Dualization with respect to subgroup generated by $S_{2,12} \ldots \ldots \ldots$

5 Duals with respect to three-dimensional subgroups 13

5.1 Dualization with respect to subgroup generated by $S_{3,2} \ldots \ldots$. . . 15

6 Duals with respect to four-dimensional subgroups 15

7 Conclusion 17

$\begin{array}{ll}\text { Appendix } & 19\end{array}$

\section{Introduction}

In string theory, the dynamics of a string propagating in curved background can be naturally characterized by non-linear sigma model on a manifold $M$ given by a tensor field $\mathcal{F}$ and action

$$
S_{\mathcal{F}}[\phi]=\int_{\Omega} \partial_{-} \phi^{\mu} \mathcal{F}_{\mu \nu}(\phi) \partial_{+} \phi^{\nu} d \xi_{+} d \xi_{-} .
$$

The symmetric and antisymmetric part of the tensor $\mathcal{F}$ represent metric $\mathcal{E}$ and torsion potential $\mathcal{B}$, called also Kalb-Ramond field or simply $B$-field, of the sigma model background.

One may apply Abelian [1] or non-Abelian [2] T-duality transformation to the sigma model (1) and obtain the dual sigma model whenever there is a Lie group $G$ generated by Killing vectors $K_{a}, a=1, \ldots, \operatorname{dim} G$ whose action is free and leaves the tensor field $\mathcal{F}$ invariant. (Non-)Abelian T-duality turns out to be a special case of Poisson-Lie Tduality [3] and it is well-known that duals exist even for backgrounds without isometries. Nevertheless, despite the fact that the geometry behind T-duality is studied thoroughly (see e.g. [4, 5] for the formulation in terms of Courant algebroids), the presence of symmetries remains crucial if one wants to dualize a particular background [6].

Renewed interest in (non-)Abelian T-duality was ignited by papers [7, 8], where it was extended to sigma models with non-vanishing Ramond fluxes, thus allowing to search for new supergravity solutions [9, 10. A special pp-wave/SYM duality is reviewed in [11. As the underlying structure accommodating T-duality is the Drinfeld double, it is also frequently used in the study of integrable sigma models and their deformations, see e.g. [12, 13] and references therein. However, our interest is slightly different.

Finding solution of equations of motion

$$
\left(\mathcal{F}_{\mu \nu}+\mathcal{F}_{\nu \mu}\right) \partial_{+} \partial_{-} \phi^{\nu}+\left(\mathcal{F}_{\mu \nu, \beta}+\mathcal{F}_{\beta \mu, \nu}-\mathcal{F}_{\beta \nu, \mu}\right) \partial_{-} \phi^{\beta} \partial_{+} \phi^{\nu}=0
$$

of a sigma model in non-trivial background is usually quite complicated and every solvable case attracts considerable attention. On the other hand, equations of motion of a sigma model in flat torsionless background are easy to solve. As the dual model may have prominently different curvature properties, one may utilize T-duality when searching 
for non-trivial solvable sigma models. Solutions of such mutually dual models are then related by T-duality transformation.

In previous papers [14] and [15], we focused on the sigma model in background given by flat four-dimensional metric, and classified its T-duals with respect to one-, two-, three-, and four-dimensional subgroups of Poincaré group given in [16]. The duals were found using (non-)Abelian T-duality with and without spectators. In both cases, we have considered the flat background without $B$-field. However, besides such model, it makes sense to dualize sigma models in backgrounds with flat metric and non-zero, but torsionless $B$-field as the bulk equations of motion (2) remain d'Alembert wave equations 1 .

In the following, we analyze the effects of additional $B$-field on the process of dualization and on the properties of dual backgrounds. In particular, we show that a suitable $B$-field allows us to dualize with respect to subgroups that otherwise could not be used for dualization, see [14]. It is often possible to partially eliminate the dependence of dual backgrounds on the $B$-field using coordinate or gauge transformations. We identify the relevant components of $\mathcal{B}$ and point out the results that could not be obtained with trivial $B$-field. Through the whole paper, we address only local properties of sigma model backgrounds because global aspects of (non-)Abelian T-duality [17] are still insufficiently understood and various issues remain obscure especially at the quantum level.

As the title of paper suggests, we focus on dual backgrounds having pp-wave metric. These are often studied in context of string theory. Backgrounds of the pp-wave form provide exact solutions to string beta equations [18, 19] and frequently emerge from WZW models [18, 20]. The ability to solve classical string equations allowed authors of papers [21, 22] to quantize the string propagating in certain classes of pp-wave backgrounds. Last but not least, pp-waves and their non-Abelian T-duals were investigated in context of supergravity e.g. in Refs. [23, 24, 25].

The structure of paper is the following. In Sec. 2, we review necessary facts concerning the dualization procedure and pp-waves. Besides, we investigate thoroughly which parts of the torsionless $B$-field affect dual backgrounds. In Secs. 3, 4, 5, and 6, we find particular pp-waves as duals of the flat torsionless background with emphasis on the cases where the addition of $B$-field provides new pp-waves. Dual pp-waves obtained before in Refs. [14, 15] without $B$-field are summarized in the Appendix.

\section{Theoretical background}

\subsection{Dualization with $B$-field}

A thorough discussion concerning the process of dualization and the differences between atomic T-duality and T-duality with spectators has been given in [14]. Therefore, we do not repeat the details, but we focus on the study of (non-)Abelian T-duality in the presence of non-zero, but torsionless $B$-field.

Consider sigma model (11) on four-dimensional target manifold $M$ for which there is a group of isometries represented by the Lie group $G$ with free action on $M$. The action of $G$ is transitive on its orbits, hence we may locally consider $M \approx(M / G) \times G$ and

\footnotetext{
${ }^{1}$ Although, the $B$-field may appear in boundary conditions for open strings.
} 
introduce adapted coordinates

$$
x^{\mu}=\left\{s^{\delta}, g^{a}\right\}, \quad \delta=1, \ldots, S, \quad a=1, \ldots, \operatorname{dim} G, \quad S=4-\operatorname{dim} G
$$

where $s^{\delta}$ label the orbits of $G$ and are treated as spectators during dualization. In these coordinates, dualizable tensor $\mathcal{F}$ is defined by symmetric matrix $E_{\xi}(s)$ and antisymmetric matrix $B(s)$ as

$$
\left(\mathcal{F}_{\xi}^{B}\right)_{\mu \nu}(s, g)=e_{\mu}^{j}(g)\left(E_{\xi}(s)+B(s)\right)_{j k} e_{\nu}^{k}(g), \quad e_{\mu}^{j}(g)=\left(\begin{array}{cc}
\mathbf{1}_{S} & 0 \\
0 & e_{c}^{a}(g)
\end{array}\right)
$$

where $e_{c}^{a}(g)$ are components of the right-invariant Maurer-Cartan form $(d g) g^{-1}$.

We are interested in dualizable sigma models with flat background, where the metric (i.e. the symmetric part $\mathcal{E}$ of $\left.\mathcal{F}_{\xi}^{B}\right)$ in suitable coordinates $x^{\rho}=(t, x, y, z)$ has the form $\eta_{\rho \sigma}=\operatorname{diag}(-1,1,1,1)$, and its Killing vectors

$$
P_{0}=\partial_{t}, \quad P_{j}=\partial_{j}, \quad L_{j}=-\varepsilon_{j k l} x^{k} \partial_{l}, \quad M_{j}=-x^{j} \partial_{t}-t \partial_{j}
$$

generate the ten-dimensional Poincaré group containing $G$ as a subgroup. As derived in [14, it is not necessary to find the transformation between $(t, x, y, z)$ and the adapted coordinates to determine the flat metric in the adapted coordinates (3). Writing

$$
E_{\xi}(s)=\left(\begin{array}{cc}
E_{\alpha \beta}(s) & E_{\alpha d}(s) \\
E_{c \beta}(s) & E_{c d}(s)
\end{array}\right), \quad \alpha, \beta=1, \ldots, S, \quad c, d=1, \ldots, \operatorname{dim} G
$$

the components of the matrix $E_{\xi}(s)$ are

$$
\begin{aligned}
E_{\alpha \beta}(s) & =\frac{\partial \xi^{\rho}}{\partial s^{\alpha}}(s) \eta_{\rho \sigma} \frac{\partial \xi^{\sigma}}{\partial s^{\beta}}(s), \\
E_{\alpha d}(s) & =\frac{\partial \xi^{\rho}}{\partial s^{\alpha}}(s) \eta_{\rho \sigma} K_{d}^{\sigma}(\xi(s)), \\
E_{c \beta}(s) & =K_{c}^{\rho}(\xi(s)) \eta_{\rho \sigma} \frac{\partial \xi^{\sigma}}{\partial s^{\beta}}(s), \\
E_{c d}(s) & =K_{c}^{\rho}(\xi(s)) \eta_{\rho \sigma} K_{d}^{\sigma}(\xi(s))
\end{aligned}
$$

where $K_{a}$ denote independent Killing vectors generating the isometry group $G$, and the functions $\xi(s)$ assign the group unit of $G$ to points of orbits of $G$ in $M$. As suggested in [26], $\xi(s)$ can be chosen arbitrarily up to certain conditions. For practical reasons, we choose these functions in accordance with [14.

The matrix

$$
B(s)=\left(\begin{array}{cc}
B_{\alpha \beta}(s) & B_{\alpha d}(s) \\
B_{c \beta}(s) & B_{c d}(s)
\end{array}\right)=\left(\begin{array}{cccc}
0 & B_{1}(s) & B_{2}(s) & B_{3}(s) \\
-B_{1}(s) & 0 & B_{4}(s) & B_{5}(s) \\
-B_{2}(s) & -B_{4}(s) & 0 & B_{6}(s) \\
-B_{3}(s) & -B_{5}(s) & -B_{6}(s) & 0
\end{array}\right)
$$

generates $G$-invariant $B$-field (i.e. the antisymmetric part $\mathcal{B}$ of $\mathcal{F}_{\xi}^{B}$ ) and torsion $\mathcal{H}=d \mathcal{B}$ via

$$
\mathcal{B}=\frac{1}{2} \mathcal{B}_{\mu \nu}(s, g) d x^{\mu} \wedge d x^{\nu}, \quad \mathcal{B}_{\mu \nu}(s, g)=e_{\mu}^{j}(g) B(s)_{j k} e_{\nu}^{k}(g) .
$$


Vanishing of the torsion restricts $B(s)$ in a way that depends on the choice of the Lie group $G$ with respect to which we dualize.

In case of (non-)Abelian T-duality, the dual tensor can be written as

$$
\widehat{\mathcal{F}}_{\xi}^{B}(s, \hat{g})=\left(\mathbf{1}+\widehat{E}_{\xi}^{B}(s) \cdot \widehat{\Pi}(\hat{g})\right)^{-1} \cdot \widehat{E}_{\xi}^{B}(s)
$$

with matrix $\widehat{E}_{\xi}^{B}(s)$ given by

$$
\widehat{E}_{\xi}^{B}(s)=\left(D_{1}+\left(E_{\xi}(s)+B(s)\right) \cdot D_{2}\right)^{-1} \cdot\left(D_{2}+\left(E_{\xi}(s)+B(s)\right) \cdot D_{1}\right)
$$

where

$$
D_{1}=\left(\begin{array}{cc}
\mathbf{1}_{S} & 0 \\
0 & \mathbf{O}_{G}
\end{array}\right), \quad D_{2}=\left(\begin{array}{cc}
\mathbf{O}_{S} & 0 \\
0 & \mathbf{1}_{G}
\end{array}\right)
$$

and

$$
\widehat{\Pi}(\hat{g})=\left(\begin{array}{cc}
\mathbf{O}_{S} & 0 \\
0 & -f_{c d}^{b} \hat{g}_{b}
\end{array}\right)
$$

where $f_{c d}{ }^{b}$ are structure coefficients of the Lie algebra of the group $G$ and $\hat{g}_{b}$ are coordinates of the Abelian group $\widehat{G}$.

As the first factor of $\widehat{E}_{\xi}^{B}(s)$ in (11) must have non-vanishing determinant, we get from (6), (8), and (12) condition of dualizability in the form

$$
\operatorname{det}\left(D_{1}+\left(E_{\xi}(s)+B(s)\right) \cdot D_{2}\right)=\operatorname{det}\left(E_{c d}(s)+B_{c d}(s)\right) \neq 0 .
$$

Compared with the case without $B$-field, the condition (14) can be satisfied even for subgroups with vanishing det $\left(E_{c d}(s)\right)$ labeled as not dualizable in [14. Similar observation was made by Borsato and Wulff in the context of deformed T-dual models [13. Consequently, the addition of advisable $B$-field may extend the number of dualizable backgrounds 2

Using formulas (10), (11), and (13), we gain dual tensors with symmetric and antisymmetric part that can be interpreted as dual metric and dual torsion potential.

\section{$2.2 \quad$ Reduction of $B$-field}

The dual backgrounds (10) may be very complicated in general. Nevertheless, the dependence of dual metric and torsion on the initial $B$-field can be often eliminated or simplified by coordinate or gauge transformation.

The initial background is described by the tensor (44) that can be written as

$$
\begin{aligned}
\mathcal{F}_{\xi}^{B}(s, g) & =\left(\begin{array}{cc}
\mathcal{F}_{\alpha \beta}(s, g) & \mathcal{F}_{\alpha d}(s, g) \\
\mathcal{F}_{c \beta}(s, g) & \mathcal{F}_{c d}(s, g)
\end{array}\right)=\left(\begin{array}{cc}
\mathcal{E}_{\alpha \beta}(s, g)+\mathcal{B}_{\alpha \beta}(s, g) & \mathcal{E}_{\alpha d}(s, g)+\mathcal{B}_{\alpha d}(s, g) \\
\mathcal{E}_{c \beta}(s, g)+\mathcal{B}_{c \beta}(s, g) & \mathcal{E}_{c d}(s, g)+\mathcal{B}_{c d}(s, g)
\end{array}\right) \\
& =\left(\begin{array}{cc}
\delta_{\alpha}^{\iota} & 0 \\
0 & e_{c}^{a}(g)
\end{array}\right) \cdot\left(\begin{array}{cc}
E_{\iota \kappa}(s)+B_{\iota \kappa}(s) & E_{\iota b}(s)+B_{\iota b}(s) \\
E_{a \kappa}(s)+B_{a \kappa}(s) & E_{a b}(s)+B_{a b}(s)
\end{array}\right) \cdot\left(\begin{array}{cc}
\delta_{\beta}^{\kappa} & 0 \\
0 & e_{d}^{b}(g)
\end{array}\right) .
\end{aligned}
$$

\footnotetext{
${ }^{2}$ Unfortunately, this does not work for one-dimensional subgroups where $B_{c d}(s)$ vanishes identically. However, it involves only one subgroup $S_{1,5}$.
} 
Vanishing of initial torsion $d \mathcal{B}=0$ implies

$$
\begin{aligned}
\partial_{[\alpha} \mathcal{B}_{\beta \gamma]} & =0, & \partial_{[\alpha} \mathcal{B}_{\beta] c}+\partial_{c} \mathcal{B}_{\alpha \beta} & =0, \\
\partial_{\alpha} \mathcal{B}_{b c}+\partial_{[b} \mathcal{B}_{c] \alpha} & =0, & \partial_{[a} \mathcal{B}_{b c]} & =0
\end{aligned}
$$

where $X_{[A B]}:=\frac{1}{2}\left(X_{A B}-X_{B A}\right)$ and $X_{[A B C]}:=\frac{1}{3 !}\left(X_{A B C}-X_{B A C} \pm \ldots\right)$. Using (15), we can rewrite (16) as

$$
\partial_{[\alpha} B_{\beta \gamma]}=0, \quad \partial_{[\alpha} B_{\beta] c}=0 .
$$

The latter condition in (18) can be (at least locally) satisfied by

$$
B_{\alpha d}(s)=\partial_{\alpha} A_{d}(s)
$$

Using (15) and Maurer-Cartan equation

$$
\partial_{c} e_{d}^{a}-\partial_{d} e_{c}^{a}-f_{j k}^{a} e_{c}^{j} e_{d}^{k}=0
$$

we can rewrite the former condition in (17) as

$$
\partial_{\alpha} B_{c d}=f_{c d}^{b} B_{\alpha b}
$$

Together with (19) we get

$$
B_{c d}(s)=b_{c d}+f_{c d}^{b} A_{b}(s)
$$

It is possible to derive general coordinate transformations in the dual manifold that eliminate some components of the torsionless $B$-field from the dual background. Thus, we can neglect these components and reduce the initial $B$-field.

For better readability, we can introduce the block form of (15) in the following way

$$
\mathcal{F}_{\xi}^{B}(s, 0)=\left(\begin{array}{ll}
F_{S S} & F_{S G} \\
F_{G S} & F_{G G}
\end{array}\right)=\left(\begin{array}{cc}
E_{S S}+B_{S S} & E_{S G}+B_{S G} \\
\left(E_{S G}\right)^{T}-\left(B_{S G}\right)^{T} & E_{G G}+B_{G G}
\end{array}\right)
$$

Matrix of components of the dual tensor obtained from the equations (10)-(13) and (23) can be written in the block form as

$$
\widehat{\mathcal{F}}_{\xi}^{B}(s, \hat{g})=\left(\begin{array}{cc}
\mathbf{1}_{S} & -F_{S G} \\
0 & \mathbf{1}_{G}
\end{array}\right) \cdot\left(\begin{array}{cc}
F_{S S} & 0 \\
0 & {\left[F_{G G}+\widehat{\Pi}_{G G}\right]^{-1}}
\end{array}\right) \cdot\left(\begin{array}{cc}
\mathbf{1}_{S} & 0 \\
F_{G S} & \mathbf{1}_{G}
\end{array}\right)
$$

where $\left(\widehat{\Pi}_{G G}\right)_{c d}=-f_{c d}{ }^{b} \hat{g}_{b}$. Using the coordinate transformation

$$
s^{\alpha}=s^{\prime \alpha}, \quad \hat{g}_{c}=\hat{g}_{c}^{\prime}+\Gamma_{c}\left(s^{\prime}\right),
$$

we obtain the dual tensor (24) in the coordinates $\left(s^{\prime}, \hat{g}^{\prime}\right)$ as

$$
\left(\widehat{\mathcal{F}}_{\xi}^{B}\right)^{\prime}\left(s^{\prime}, \hat{g}^{\prime}\right)=\left(\begin{array}{cc}
\mathbf{1}_{S} & \partial_{S} \Gamma_{G} \\
0 & \mathbf{1}_{G}
\end{array}\right) \cdot\left(\begin{array}{cc}
\widehat{\mathcal{F}}_{S S} & \widehat{\mathcal{F}}_{S G} \\
\widehat{\mathcal{F}}_{G S} & \widehat{\mathcal{F}}_{G G}
\end{array}\right) \cdot\left(\begin{array}{cc}
\mathbf{1}_{S} & 0 \\
\left(\partial_{S} \Gamma_{G}\right)^{T} & \mathbf{1}_{G}
\end{array}\right)=
$$




$$
=\left(\begin{array}{cc}
\mathbf{1}_{S} & \partial_{S} \Gamma_{G}-F_{S G} \\
0 & \mathbf{1}_{G}
\end{array}\right) \cdot\left(\begin{array}{cc}
F_{S S} & 0 \\
0 & {\left[F_{G G}+\widehat{\Pi}_{G G}^{\prime}\right]^{-1}}
\end{array}\right) \cdot\left(\begin{array}{cc}
\mathbf{1}_{S} & 0 \\
\left(\partial_{S} \Gamma_{G}\right)^{T}+F_{G S} & \mathbf{1}_{G}
\end{array}\right),
$$

where $\left(\partial_{S} \Gamma_{G}\right)_{\alpha d}=\partial_{\alpha} \Gamma_{d}\left(s^{\prime}\right)$ and $\left(\widehat{\Pi}_{G G}^{\prime}\right)_{c d}=-f_{c d}{ }^{b}\left(\hat{g}_{b}^{\prime}+\Gamma_{b}\left(s^{\prime}\right)\right)$. It is clear from (19), (22), and (25) that by the choice $\Gamma_{c}\left(s^{\prime}\right)=A_{c}\left(s^{\prime}\right)$, we can eliminate the dependence of the dual tensor (26) on $A_{c}\left(s^{\prime}\right)$ by the coordinate transformation. Besides, the dual torsion is not influenced by $B_{S S}$ due to the former condition in (18) and consequently, we can eliminate the dependence of the dual tensor (26) on $B_{\alpha \beta}\left(s^{\prime}\right)$ by a gauge transformation.

As result, we can assume without loss of generality that functions $A_{c}(s)$ and $B_{\alpha \beta}(s)$ vanish. Using (19) and (22), we then get $B_{\alpha d}(s)=0$ and $B_{c d}(s)=b_{c d}=$ const. It means that if we are interested in dual backgrounds only up to coordinate and gauge transformations, we can restrict ourselves to initial B-fields given by the constant matrix

$$
B(s)=\left(\begin{array}{cc}
\mathbf{O}_{S} & 0 \\
0 & B_{c d}
\end{array}\right), \quad B_{c d}=b_{c d}=\text { const }
$$

The dual tensor (24) then reads

$$
\widehat{\mathcal{F}}_{\xi}^{B}(s, \hat{g})=\left(\begin{array}{cc}
\mathbf{1}_{S} & -E_{S G} \\
0 & \mathbf{1}_{G}
\end{array}\right) \cdot\left(\begin{array}{cc}
E_{S S} & 0 \\
0 & {\left[E_{G G}+B_{G G}+\widehat{\Pi}_{G G}\right]^{-1}}
\end{array}\right) \cdot\left(\begin{array}{cc}
\mathbf{1}_{S} & 0 \\
\left(E_{S G}\right)^{T} & \mathbf{1}_{G}
\end{array}\right),
$$

where $\left(B_{G G}\right)_{c d}=B_{c d}$. Owing to (20) and (27), the latter condition in (17) can be rewritten as

$$
\partial_{[a} \mathcal{B}_{b c]}=\left(\partial_{[a} e_{b}^{d}\right) e_{c]}^{h} B_{d h}=-e_{[a}^{j} e_{b}^{k} e_{c]}^{h} f_{j k}^{d} B_{d h}=0,
$$

resulting in the restricting condition for the constants $B_{c d}$ in the form

$$
f_{[a b}^{d} B_{c] d}=0 .
$$

Finally, let us emphasize that considerations made in this section are valid for arbitrary dimensions of $M$ and $G$ as well as for arbitrary initial metric. Constants $B_{c d}$ satisfying (29) can be considered as components of a 2-cocycle of Lie algebra of the group $G$. Therefore, addition of the torsionless $B$-field to the initial background can always be interpreted as the application of the deformed T-duality [12] 3

\subsection{Plane-parallel waves}

In this paper, we restrict ourselves to duals of the flat torsionless background whose metrics are the so-called plane-parallel (pp-)waves.

In general, pp-wave metrics admit covariantly constant null Killing vector and exhibit particularly simple curvature properties since their scalar curvature vanishes and in suitable coordinates the Ricci tensor has only one non-zero component.

All pp-wave metrics obtained below can be expressed in the Brinkmann coordinates as

$$
d s^{2}=\left(K_{3}(u) z_{3}^{2}+K_{4}(u) z_{4}^{2}\right) d u^{2}+2 d u d v+d z_{3}^{2}+d z_{4}^{2},
$$

\footnotetext{
${ }^{3}$ We are gratefull to the authors of [12] for pointing out this fact.
} 
or the Rosen coordinates as

$$
d s^{2}=2 d u d v+C_{1}(u) d x_{1}^{2}+C_{2}(u) d x_{2}^{2}
$$

In this case, there is rather simple relation between these two forms (see e.g. [27])

$$
\begin{aligned}
C_{1}(u) & =Q_{1}(u)^{2}, & K_{3}(u) & =\frac{1}{Q_{1}(u)} \frac{d^{2} Q_{1}}{d u^{2}}(u), \\
C_{2}(u) & =Q_{2}(u)^{2}, & K_{4}(u) & =\frac{1}{Q_{2}(u)} \frac{d^{2} Q_{2}}{d u^{2}}(u) .
\end{aligned}
$$

As one can see, the correspondence is not one-to-one because for given $K_{3}(u)$ and $K_{4}(u)$ the functions $Q_{1}(u)$ and $Q_{2}(u)$ are solutions of linear differential equations of the second order. In other words, the Rosen form of metric is not unique in general.

These pp-waves have the form of the Penrose-Güven limit [28, 29] with metric (30) and torsion

$$
H=H(u) d u \wedge d z_{3} \wedge d z_{4} .
$$

The one-loop conformal invariance conditions in this case simplify substantially to solvable differential equation for the dilaton $\Phi=\Phi(u)$

$$
\Phi^{\prime \prime}(u)+K_{3}(u)+K_{4}(u)+\frac{1}{2} H(u)^{2}=0 .
$$

Special pp-wave backgrounds obtained in Refs. [18, 20] from gauged WZW models are given (up to gauge transformation of the $B$-field) in the Rosen coordinates as

$$
\begin{gathered}
d s^{2}=d u d v+\frac{g_{1}\left(u^{\prime}\right)}{g_{1}\left(u^{\prime}\right) g_{2}(u)+q^{2}} d x_{1}^{2}+\frac{g_{2}(u)}{g_{1}\left(u^{\prime}\right) g_{2}(u)+q^{2}} d x_{2}^{2}, \\
\mathcal{B}_{12}\left(u, v, x_{1}, x_{2}\right)=\frac{q}{g_{1}\left(u^{\prime}\right) g_{2}(u)+q^{2}}
\end{gathered}
$$

with the Sfetsos-Tseytlin parameters $q=$ const, $u^{\prime}=a u+d \quad(a, d=$ const $)$ and $g_{i}(u)$ taking any of the following forms

$$
\left\{1, u^{2}, \tanh ^{2} u, \tan ^{2} u, u^{-2}, \operatorname{coth}^{2} u, \cot ^{2} u\right\} \text {. }
$$

Several of these pp-waves have been already discovered in Refs. [14] and [15] as duals of the flat background. As we shall see, dualization with non-zero, but torsionless $B$-fields yields also other cases of (36). Exact string solutions from gauged WZW models in the form of plane gravitational waves and their duals are investigated also in the paper [30.

In the following, we focus on duals of the flat torsionless backgrounds exhibiting ppwave characteristics. Especially, we attend to subgroups that provide new pp-waves not found in [14, 15] and find their Brinkmann and Rosen forms with the Sfetsos-Tseytlin parameters $g_{1}(u), g_{2}(u)$, and $q$. We also cover some other attractive cases that demonstrate general ideas developed in the previous section. Discussion of pp-waves that appeared already in [14, 15] without presence of the $B$-field is included in the Appendix. 


\begin{tabular}{|c||c|c|}
\hline & Generators & Free and transitive action for \\
\hline \hline$S_{1,1}$ & $\begin{array}{c}\cos \gamma L_{3}+\sin \gamma M_{3}, \\
0<\gamma<\pi, \gamma \neq \frac{\pi}{2}\end{array}$ & $\begin{array}{c}t \neq 0 \vee x \neq 0 \vee \\
\vee y \neq 0 \vee z \neq 0\end{array}$ \\
\hline$S_{1,2}$ & $L_{3}$ & $x \neq 0 \vee y \neq 0$ \\
\hline$S_{1,3}$ & $M_{3}$ & $t \neq 0 \vee z \neq 0$ \\
\hline$S_{1,4}$ & $L_{2}+M_{1}$ & $x \neq 0 \vee t+z \neq 0$ \\
\hline$S_{1,5}$ & $P_{0}-P_{3}$ & $\mathbb{R}^{4}$ \\
\hline$S_{1,6}$ & $P_{3}$ & $\mathbb{R}^{4}$ \\
\hline$S_{1,7}$ & $P_{0}$ & $\mathbb{R}^{4}$ \\
\hline$S_{1,8}$ & $L_{3}+\epsilon\left(P_{0}+P_{3}\right)$, & $\mathbb{R}^{4}$ \\
\hline$S_{1,9}$ & $\epsilon= \pm 1$ & $\mathbb{R}^{4}$ \\
\hline$S_{1,10}$ & $L_{3}+\alpha P_{0}, \quad \alpha>0$ & $\mathbb{R}^{4}$ \\
\hline$S_{1,11} \quad \alpha \neq 0$ & $M_{3}+\alpha P_{1}, \quad \alpha>0$ & $\mathbb{R}^{4}$ \\
\hline$S_{1,12}$ & $L_{2}+M_{1}+P_{0}+P_{3}$ & $\mathbb{R}^{4}$ \\
\hline$S_{1,13}$ & $L_{2}+M_{1}+\epsilon P_{2}$, & $\mathbb{R}^{4}$ \\
\hline
\end{tabular}

Table 1: Generators of one-dimensional subgroups of the Poincaré group with open subsets of transitive and free action

\section{Duals with respect to one-dimensional subgroups}

It is clear from above that torsionless initial backgrounds do not influence the T-duals with respect to the one-dimensional subgroups as $B_{c d}=0$. Indeed, a torsionless background on $M$ is obtained if the functions $B_{i}\left(s_{1}, s_{2}, s_{3}\right), i=1, \ldots, 6$ fulfill conditions (cf.(16)) and (17))

$$
\begin{gathered}
\partial_{s_{3}} B_{1}\left(s_{1}, s_{2}, s_{3}\right)-\partial_{s_{2}} B_{2}\left(s_{1}, s_{2}, s_{3}\right)+\partial_{s_{1}} B_{4}\left(s_{1}, s_{2}, s_{3}\right)=0, \\
\partial_{s_{1}} B_{5}\left(s_{1}, s_{2}, s_{3}\right)-\partial_{s_{2}} B_{3}\left(s_{1}, s_{2}, s_{3}\right)=0 \\
\partial_{s_{1}} B_{6}\left(s_{1}, s_{2}, s_{3}\right)-\partial_{s_{3}} B_{3}\left(s_{1}, s_{2}, s_{3}\right)=0 \\
\partial_{s_{2}} B_{6}\left(s_{1}, s_{2}, s_{3}\right)-\partial_{s_{3}} B_{5}\left(s_{1}, s_{2}, s_{3}\right)=0 .
\end{gathered}
$$

However, results of the Sec. 2.2 imply that we can eliminate $B_{3}, B_{5}, B_{6}$ by the coordinate transformation (25) and $B_{1}, B_{2}, B_{4}$ by a gauge transformation. It means that addition of torsionless $B$-field does not influence the duals with respect to one-dimensional subgroups obtained in [14].

Depending on the particular choice of Killing vector generating group $G$, we get various dual backgrounds. Duals obtained with respect to subgroups generated by (see Tab. 1) $S_{1,1}, S_{1,2}, S_{1,3}, S_{1,8}, S_{1,9}, S_{1,10}, S_{1,11}, S_{1,12}$ do not represent pp-waves since their scalar curvatures do not vanish. The initial background cannot be dualized with respect to subgroup generated by $S_{1,5}$ because even after addition of antisymmetric $B$-field the condition of dualizability (14) is not satisfied.

Duals obtained with respect to subgroups generated by $S_{1,6}, S_{1,7}$ with $B$-field satisfying conditions (38) and (39) become flat backgrounds with vanishing torsion. The only pp-waves obtained as duals of the flat metric and torsionless $B$-field might appear via 
dualization with respect to subgroups generated by $S_{1,4}$ and $S_{1,13}$. Their form was already given in [14].

We should emphasize that when the $B$-field is not torsionless, the dual backgrounds may have completely different properties. As an example, let us consider the case of $S_{1,4}$. If (38) does not hold, the dual background is a pp-wave with torsion. If (39) is violated, the scalar curvature of the dual metric does not vanish, and it is no longer a pp-wave.

\section{Duals with respect to two-dimensional subgroups}

Results of Sec. 2.2 imply that we can eliminate $B_{2}, \ldots, B_{5}$ by the coordinate transformation (25) and $B_{1}$ by a gauge transformation. Therefore, the only relevant component is the constant $B_{6}$ satisfying (29).

Subgroups generated by

$$
S_{2,3}, S_{2,7}, S_{2,9}, S_{2,18}
$$

can be used for dualization only with $B_{6} \neq 0$ because the condition (14) for these subgroups reads

$$
\operatorname{det}\left(E_{c d}(s)+B_{c d}(s)\right)=B_{6}^{2} \neq 0 .
$$

Setting $B_{6}=0$ in the other cases restores the results of [14].

Duals obtained with respect to subgroups generated by $S_{2,1}, S_{2,4}, S_{2,5}, S_{2,6}, S_{2,14}$, $S_{2,15}, S_{2,16}, S_{2,19}, S_{2,20}$, and $S_{2,23}$ do not represent pp-waves as their scalar curvatures do not vanish for any $B_{6}$. Duals obtained with respect to subgroups generated by $S_{2,7}, S_{2,9}$, $S_{2,10}, S_{2,11}, S_{2,17}, S_{2,18}, S_{2,22}$, and $S_{2,24}$ and torsionless $B$-field are flat backgrounds with vanishing torsion.

The other dual backgrounds are pp-waves. Nevertheless, the duals obtained via $S_{2,3}$, $S_{2,8}, S_{2,13}$, and $S_{2,21}$ reproduce pp-waves found previously without $B$-field. Thus, we defer the discussion of these backgrounds to the Appendix with the exception of the interesting case of $S_{2,3}$.

Below, we present duals with respect to subgroups generated by $S_{2,2}$ and $S_{2,12}$ where the introduction of $B$-field results in new pp-waves and the special case of $S_{2,3}$ that yields pp-wave only for a suitable $B$-field.

\subsection{Dualization with respect to subgroup generated by $S_{2,2}$}

Similarly to [14], we choose $\xi(s)=\left(-\frac{s_{2}}{2 s_{1}}, 0,0, s_{1}+\frac{s_{2}}{2 s_{1}}\right)$ and get the dual background in the form

$$
\widehat{\mathcal{F}}(s, \hat{g})=\left(\begin{array}{cccc}
1-\frac{s_{2}}{s_{1}^{2}} & \frac{1}{2 s_{1}} & 0 & 0 \\
\frac{1}{2 s_{1}} & 0 & 0 & 0 \\
0 & 0 & \frac{s_{1}^{2}}{s_{1}^{4}+B_{6}^{2}} & -\frac{B_{6}}{s_{1}^{4}+B_{6}^{2}} \\
0 & 0 & \frac{B_{6}}{s_{1}^{4}+B_{6}^{2}} & \frac{s_{1}^{2}}{s_{1}^{4}+B_{6}^{2}}
\end{array}\right) .
$$




\begin{tabular}{|c|c|c|}
\hline & Generators & Free and transitive action for \\
\hline$S_{2,1}$ & $L_{3}, M_{3}$ & $\begin{array}{l}y z \neq 0 \vee x z \neq 0 \vee \\
\vee t y \neq 0 \vee t x \neq 0\end{array}$ \\
\hline$S_{2,2}$ & $L_{2}+M_{1}, L_{1}-M_{2}$ & $t+z \neq 0$ \\
\hline$S_{2,3}$ & $L_{3}, P_{0}-P_{3}$ & $x \neq 0 \vee y \neq 0$ \\
\hline$S_{2,4}$ & $L_{3}, P_{3}$ & $x \neq 0 \vee y \neq 0$ \\
\hline$S_{2,5}$ & $L_{3}, P_{0}$ & $x \neq 0 \vee y \neq 0$ \\
\hline$S_{2,6}$ & $M_{3}, P_{1}$ & $t \neq 0 \vee z \neq 0$ \\
\hline$S_{2,7}$ & $L_{2}+M_{1}, P_{0}-P_{3}$ & $t+z \neq 0$ \\
\hline$S_{2,8}$ & $L_{2}+M_{1}, P_{2}$ & $t+z \neq 0 \vee x \neq 0$ \\
\hline$S_{2,9}$ & $P_{0}-P_{3}, P_{1}$ & $\mathbb{R}^{4}$ \\
\hline$S_{2,10}$ & $P_{0}, P_{3}$ & $\mathbb{R}^{4}$ \\
\hline$S_{2,11}$ & $P_{1}, P_{2}$ & $\mathbb{R}^{4}$ \\
\hline$S_{2,12}$ & $L_{2}+M_{1}, L_{1}-M_{2}+P_{2}$ & $\begin{array}{c}y(t+z) \neq 0 \vee \\
\vee x(1+t+z) \neq 0 \vee \\
\vee(t+z)(1+t+z) \neq 0\end{array}$ \\
\hline$S_{2,13}$ & $\begin{array}{c}L_{3}+\epsilon\left(P_{0}+P_{3}\right), P_{0}-P_{3}, \\
\quad \epsilon= \pm 1\end{array}$ & $\mathbb{R}^{4}$ \\
\hline$S_{2,14}$ & $L_{3}+\alpha P_{0}, P_{3}, \quad \alpha>0$ & $\mathbb{R}^{4}$ \\
\hline$S_{2,15}$ & $L_{3}+\alpha P_{3}, P_{0}, \quad \alpha \neq 0$ & $\mathbb{R}^{4}$ \\
\hline$S_{2,16}$ & $M_{3}+\alpha P_{2}, P_{1}, \quad \alpha>0$ & $\mathbb{R}^{4}$ \\
\hline$S_{2,17}$ & $L_{2}+M_{1}+P_{0}+P_{3}, P_{0}-P_{3}$ & $\mathbb{R}^{4}$ \\
\hline$S_{2,18}$ & $\begin{array}{c}L_{2}+M_{1}+\epsilon P_{2}, P_{0}-P_{3} \\
\epsilon= \pm 1\end{array}$ & $\mathbb{R}^{4}$ \\
\hline$S_{2,19}$ & $L_{2}+M_{1}+P_{0}+P_{3}, P_{2}$ & $\mathbb{R}^{4}$ \\
\hline$S_{2,20}$ & $M_{3}, L_{2}+M_{1}$ & $\begin{array}{c}z(t+z) \neq 0 \vee \\
\vee x(t+z) \neq 0 \vee \\
\vee t(t+z) \neq 0\end{array}$ \\
\hline$S_{2,21}$ & $\begin{array}{c}\cos \gamma L_{3}+\sin \gamma M_{3}, P_{0}-P_{3} \\
0<\gamma<\pi, \gamma \neq \frac{\pi}{2}\end{array}$ & $\begin{array}{c}t+z \neq 0 \vee \\
\vee x \neq 0 \vee y \neq 0\end{array}$ \\
\hline$S_{2,22}$ & $M_{3}, P_{0}-P_{3}$ & $t+z \neq 0$ \\
\hline$S_{2,23}$ & $M_{3}+\alpha P_{2}, L_{2}+M_{1}, \quad \alpha>0$ & $t+z \neq 0 \vee x \neq 0$ \\
\hline$S_{2,24}$ & $M_{3}+\alpha P_{2}, P_{0}-P_{3}, \quad \alpha>0$ & $\mathbb{R}^{4}$ \\
\hline
\end{tabular}

Table 2: Generators of two-dimensional subgroups of the Poincaré group with open subsets of transitive and free action 
Subsequent coordinate transformation

$$
\begin{array}{ll}
s_{1}=u, & \hat{g}_{1}=\frac{z_{3} \sqrt{B_{6}^{2}+u^{4}}}{u}, \\
s_{2}=-u^{2}+2 u v+\frac{\left(B_{6}^{2}-u^{4}\right)\left(z_{3}^{2}+z_{4}^{2}\right)}{B_{6}^{2}+u^{4}}, & \hat{g}_{2}=\frac{z_{4} \sqrt{B_{6}^{2}+u^{4}}}{u}
\end{array}
$$

brings the dual metric to the Brinkmann form

$$
d s^{2}=\frac{2 u^{2}\left(u^{4}-5 B_{6}^{2}\right)}{\left(u^{4}+B_{6}^{2}\right)^{2}}\left(z_{3}^{2}+z_{4}^{2}\right) d u^{2}+2 d u d v+d z_{3}^{2}+d z_{4}^{2} .
$$

The corresponding torsion of this model is

$$
\widehat{\mathcal{H}}=\frac{4 B_{6} u}{u^{4}+B_{6}^{2}} d u \wedge d z_{3} \wedge d z_{4} .
$$

The Rosen form of the metric is

$$
d s^{2}=2 d u d v+\frac{u^{2}}{u^{4}+B_{6}^{2}}\left(d x_{1}^{2}+d x_{2}^{2}\right)
$$

that corresponds to the Sfetsos-Tseytlin parameters $g_{1}(u)=g_{2}(u)=u^{2}$ and $q=B_{6}$. This restores the result of [14] for $B_{6}=0$. For $B_{6} \neq 0$ we get new pp-wave. One can then perform additional change of coordinates that effectively rescales $B_{6}$ to 1 .

\subsection{Dualization with respect to subgroup generated by $S_{2,3}$}

This is one of the cases where dualization is possible only for $B_{6} \neq 0$. We choose $\xi(s)=$ $\left(\frac{s_{1}}{2}, s_{2}, 0, \frac{s_{1}}{2}\right)$ and get the dual background in the simple form

$$
\widehat{\mathcal{F}}(s, \hat{g})=\left(\begin{array}{cccc}
-\frac{s_{2}^{2}}{B_{6}^{2}}-1 & 0 & \frac{1}{B_{6}} & \frac{s_{2}^{2}}{B_{6}^{2}} \\
0 & 1 & 0 & 0 \\
\frac{1}{B_{6}} & 0 & 0 & -\frac{1}{B_{6}} \\
-\frac{s_{2}^{2}}{B_{6}^{2}} & 0 & \frac{1}{B_{6}} & \frac{s_{2}^{2}}{B_{6}^{2}}
\end{array}\right) .
$$

The coordinate transformation

$$
s_{1}=B_{6} u, \quad s_{2}=\sqrt{z_{3}^{2}+z_{4}^{2}}, \quad \hat{g}_{1}=\frac{B_{6}^{2} u}{2}+v, \quad \hat{g}_{2}=B_{6} \arctan \frac{z_{4}}{z_{3}}
$$

then brings the dual metric to Brinkmann form independent of the $B$-field

$$
d s^{2}=-\left(z_{3}^{2}+z_{4}^{2}\right) d u^{2}+2 d u d v+d z_{3}^{2}+d z_{4}^{2} .
$$

The corresponding torsion is

$$
\widehat{\mathcal{H}}=2 d u \wedge d z_{3} \wedge d z_{4} .
$$

The Rosen form of the dual metric and torsion are

$$
\begin{gathered}
d s^{2}=2 d u d v+\cos ^{2} u d x_{1}^{2}+\sin ^{2} u d x_{2}^{2}, \\
\widehat{\mathcal{H}}=\sin (2 u) d u \wedge d x_{1} \wedge d x_{2} .
\end{gathered}
$$

This background appeared already in [14, 15] by dualization with respect to subgroups generated by $S_{2,13}, S_{2,21}, S_{4,17}$ and $S_{4,29}$ without $B$-field. 


\subsection{Dualization with respect to subgroup generated by $S_{2,12}$}

We choose $\xi(s)=\left(-\frac{s_{2}}{1+s_{1}}, 0,0, s_{1}+\frac{s_{2}}{1+s_{1}}\right)$ and get the dual background in the form

$$
\widehat{\mathcal{F}}(s, \hat{g})=\left(\begin{array}{cccc}
\frac{s_{1}^{2}+2 s_{1}-2 s_{2}+1}{\left(s_{1}+1\right)^{2}} & \frac{1}{s_{1}+1} & 0 & 0 \\
\frac{1}{s_{1}+1} & 0 & 0 & 0 \\
0 & 0 & \frac{\left(s_{1}+1\right)^{2}}{B_{6}^{2}+s_{1}^{2}\left(s_{1}+1\right)^{2}} & -\frac{B_{6}}{B_{6}^{2}+s_{1}^{2}\left(s_{1}+1\right)^{2}} \\
0 & 0 & \frac{B_{6}}{B_{6}^{2}+s_{1}^{2}\left(s_{1}+1\right)^{2}} & \frac{s_{1}^{2}}{B_{6}^{2}+s_{1}^{2}\left(s_{1}+1\right)^{2}}
\end{array}\right) .
$$

Subsequent coordinate transformation

$$
\begin{gathered}
s_{1}=u \\
s_{2}=\frac{B_{6}^{2}\left(-u^{3}+u^{2}(2 v-1)+u\left(2 v+z_{3}^{2}+z_{4}^{2}\right)+z_{4}^{2}\right)}{2 u\left(B_{6}^{2}+u^{2}(u+1)^{2}\right)} \\
-\frac{u^{2}(u+1)^{2}\left(u^{3}+u^{2}(1-2 v)+u\left(-2 v+z_{3}^{2}+z_{4}^{2}\right)+z_{3}^{2}\right)}{2 u\left(B_{6}^{2}+u^{2}(u+1)^{2}\right)}, \\
\hat{g}_{1}=\frac{z_{3} \sqrt{B_{6}^{2}+u^{2}(u+1)^{2}}}{u+1}, \quad \hat{g}_{2}=-\frac{z_{4} \sqrt{B_{6}^{2}+u^{2}(u+1)^{2}}}{u}
\end{gathered}
$$

brings this pp-wave metric to the Brinkmann form

$$
\begin{gathered}
d s^{2}=\left(\frac{2 u^{2}(u+1)^{4}-\omega^{2}(2 u(5 u+4)+1)}{\left(u^{2}(u+1)^{2}+\omega^{2}\right)^{2}} z_{3}^{2}+\frac{2 u^{4}(u+1)^{2}-\omega^{2}(2 u(5 u+6)+3)}{\left(u^{2}(u+1)^{2}+\omega^{2}\right)^{2}} z_{4}^{2}\right) d u^{2} \\
+2 d u d v+d z_{3}^{2}+d z_{4}^{2}
\end{gathered}
$$

where $\omega=B_{6}$. The corresponding torsion of this model is

$$
\widehat{\mathcal{H}}=\frac{2 \omega(2 u+1)}{u^{2}(u+1)^{2}+\omega^{2}} d u \wedge d z_{3} \wedge d z_{4} .
$$

The Rosen form of the metric is

$$
d s^{2}=2 d u d v+\frac{(1+u)^{2}}{u^{2}(1+u)^{2}+\omega^{2}} d x_{1}^{2}+\frac{u^{2}}{u^{2}(1+u)^{2}+\omega^{2}} d x_{2}^{2}
$$

that corresponds to the Sfetsos-Tseytlin parameters $g_{1}\left(u^{\prime}\right)=(1+u)^{2}, g_{2}(u)=u^{2}$, and $q=\omega$. This restores the result of [14] for $\omega=0$, but not so for general $\omega \neq 0$. Moreover, the constant $\omega$ cannot be rescaled to a fixed value and hence, we get a new one-parametric set of pp-waves dual to the flat torsionless background.

\section{Duals with respect to three-dimensional subgroups}

Results of Sec. 2.2 imply that we can eliminate $B_{1}, B_{2}$, and $B_{3}$ by the coordinate transformation (25). From Eq. (27) we find that the only relevant components are the constants $B_{4}, B_{5}$, and $B_{6}$ satisfying (29). 


\begin{tabular}{|c|c|c|}
\hline & Generators & Free and transitive action for \\
\hline$S_{3,1}$ & $L_{2}+M_{1}, L_{1}-M_{2}, P_{0}-P_{3}$ & $t+z \neq 0$ \\
\hline$S_{3,2}$ & $L_{3}, P_{0}, P_{3}$ & $x \neq 0 \vee y \neq 0$ \\
\hline$S_{3,3}$ & $M_{3}, P_{1}, P_{2}$ & $t \neq 0 \vee z \neq 0$ \\
\hline$S_{3,4}$ & $L_{2}+M_{1}, P_{0}-P_{3}, P_{2}$ & $t+z \neq 0$ \\
\hline$S_{3,5}$ & $P_{0}-P_{3}, P_{1}, P_{2}$ & $\mathbb{R}^{4}$ \\
\hline$S_{3,6}$ & $P_{1}, P_{2}, P_{3}$ & $\mathbb{R}^{4}$ \\
\hline$S_{3,7}$ & $P_{0}, P_{1}, P_{2}$ & $\mathbb{R}^{4}$ \\
\hline$S_{3,8}$ & $L_{2}+M_{1}, L_{1}-M_{2}+P_{2}, P_{0}-P_{3}$ & $(t+z)(1+t+z) \neq 0$ \\
\hline$S_{3,9}$ & $L_{2}+M_{1}-\frac{1}{2}\left(P_{0}+P_{3}\right), P_{0}-P_{3}, P_{2}$ & $\mathbb{R}^{4}$ \\
\hline$S_{3,10}$ & $M_{3}, L_{2}+M_{1}, P_{2}$ & $t+z \neq 0$ \\
\hline$S_{3,11}$ & $M_{3}, P_{0}-P_{3}, L_{3}$ & $(t+z)\left(x^{2}+y^{2}\right) \neq 0$ \\
\hline$S_{3,12}$ & $M_{3}, P_{0}-P_{3}, P_{2}$ & $t+z \neq 0$ \\
\hline$S_{3,13}$ & $M_{3}+\alpha P_{2}, P_{0}-P_{3}, P_{1}, \quad \alpha>0$ & $\mathbb{R}^{4}$ \\
\hline$S_{3,14}$ & $L_{2}+M_{1}, P_{1}, P_{0}-P_{3}$ & $\emptyset$ \\
\hline$S_{3,15}$ & $L_{2}+M_{1}, P_{2}+\beta P_{1}, P_{0}-P_{3}, \quad \beta \neq 0$ & $t+z \neq 0$ \\
\hline$S_{3,16}$ & $\begin{array}{c}L_{2}+M_{1}-\epsilon P_{2}, L_{1}-M_{2}+\beta P_{2}-\epsilon P_{1}, P_{0}-P_{3}, \\
\beta>0, \epsilon= \pm 1\end{array}$ & $\epsilon^{2}+(t+z)(\beta+t+z) \neq 0$ \\
\hline$S_{3,17}$ & $L_{2}+M_{1}-\epsilon P_{2}, L_{1}-M_{2}-\epsilon P_{1}, P_{0}-P_{3}, \quad \epsilon= \pm 1$ & $\mathbb{R}^{4}$ \\
\hline$S_{3,18}$ & $L_{2}+M_{1}-\frac{1}{2}\left(P_{0}+P_{3}\right), P_{1}, P_{0}-P_{3}$ & $\mathbb{R}^{4}$ \\
\hline$S_{3,19}$ & $L_{2}+M_{1}-\epsilon P_{2}, P_{1}, P_{0}-P_{3}, \quad \epsilon= \pm 1$ & $\mathbb{R}^{4}$ \\
\hline$S_{3,20}$ & $L_{2}+M_{1}-\frac{1}{2}\left(P_{0}+P_{3}\right), P_{2}-\beta P_{1}, P_{0}-P_{3}, \quad \beta \neq 0$ & $\mathbb{R}^{4}$ \\
\hline$S_{3,21}$ & $\begin{array}{c}L_{2}+M_{1}-\epsilon P_{2}, P_{2}-\beta P_{1}, P_{0}-P_{3} \\
\beta \neq 0, \epsilon= \pm 1\end{array}$ & $t+z+\beta \epsilon \neq 0$ \\
\hline$S_{3,22}$ & $M_{3}+\alpha P_{1}, L_{2}+M_{1}, P_{0}-P_{3}, \alpha>0$ & $t+z \neq 0$ \\
\hline$S_{3,23}$ & $\begin{array}{c}M_{3}-\alpha P_{2}+\beta P_{1}, L_{2}+M_{1}, P_{0}-P_{3} \\
\alpha>0, \beta \neq 0\end{array}$ & $t+z \neq 0$ \\
\hline$S_{3,24}$ & $M_{3}, L_{2}+M_{1}, L_{1}-M_{2}$ & $t+z \neq 0$ \\
\hline$S_{3,25}$ & $M_{3}, L_{2}+M_{1}, P_{0}-P_{3}$ & $t+z \neq 0$ \\
\hline$S_{3,26}$ & $M_{3}+\alpha P_{2}, L_{2}+M_{1}, P_{0}-P_{3}, \quad \alpha>0$ & $t+z \neq 0$ \\
\hline$S_{3,27}$ & $\cos \gamma L_{3}+\sin \gamma M_{3}, P_{0}, P_{3}, \quad 0<\gamma<\pi, \gamma \neq \frac{\pi}{2}$ & $x \neq 0 \vee y \neq 0$ \\
\hline$S_{3,28}$ & $M_{3}, P_{0}, P_{3}$ & $\emptyset$ \\
\hline$S_{3,29}$ & $M_{3}+\alpha P_{2}, P_{0}, P_{3}, \quad \alpha>0$ & $\mathbb{R}^{4}$ \\
\hline$S_{3,30}$ & $L_{3}, L_{2}+M_{1}, L_{1}-M_{2}$ & $\emptyset$ \\
\hline$S_{3,31}$ & $\cos \gamma L_{3}+\sin \gamma M_{3}, P_{1}, P_{2}, \quad 0<\gamma<\pi, \gamma \neq \frac{\pi}{2}$ & $t \neq 0 \vee z \neq 0$ \\
\hline$S_{3,32}$ & $L_{3}, P_{1}, P_{2}$ & $\emptyset$ \\
\hline$S_{3,33}$ & $L_{3}+\epsilon\left(P_{0}-P_{3}\right), L_{2}+M_{1}, L_{1}-M_{2}, \epsilon= \pm 1$ & $t+z \neq 0$ \\
\hline$S_{3,34}$ & $L_{3}-\epsilon\left(P_{0}+P_{3}\right), P_{1}, P_{2}, \epsilon= \pm 1$ & $\mathbb{R}^{4}$ \\
\hline$S_{3,35}$ & $L_{3}+\alpha P_{0}, P_{1}, P_{2}, \alpha>0$ & $\mathbb{R}^{4}$ \\
\hline$S_{3,36}$ & $L_{3}+\alpha P_{3}, P_{1}, P_{2}, \alpha \neq 0$ & $\mathbb{R}^{4}$ \\
\hline$S_{3,37}$ & $\begin{array}{c}\cos \gamma L_{3}+\sin \gamma M_{3}, L_{2}+M_{1}, L_{1}-M_{2} \\
0<\gamma<\pi, \gamma \neq \frac{\pi}{2} \\
\end{array}$ & $t+z \neq 0$ \\
\hline$S_{3,38}$ & $L_{3}, M_{1}, M_{2}$ & $\emptyset$ \\
\hline$S_{3,39}$ & $L_{1}, L_{2}, L_{3}$ & $\emptyset$ \\
\hline
\end{tabular}

Table 3: Generators of three-dimensional subgroups of the Poincaré group with open subsets of transitive and free action 
There are many subgroups, namely those generated by

$$
S_{3,1}, S_{3,4}, S_{3,5}, S_{3,8}, S_{3,15}, S_{3,16}, S_{3,17}, S_{3,19}, S_{3,21}, S_{3,33}, S_{3,34}
$$

(see Tab. 3), that cannot be used for dualization without a $B$-field since $\operatorname{det}\left(E_{c d}(s)\right)=0$ (see Tabs. 9-11 in [14]). Addition of the suitable $B$-field with vanishing torsion enables dualization.

Duals obtained with respect to subgroups generated by $S_{3,5}, S_{3,6}, S_{3,7}, S_{3,12}$, and $S_{3,13}$ with initial torsionless $B$-field are flat torsionless backgrounds. Duals obtained with respect to subgroups generated by $S_{3,3}, S_{3,9}, S_{3,10}, S_{3,11}, S_{3,18}, S_{3,20}, S_{3,24}, S_{3,29}, S_{3,31}$, and $S_{3,33}, \ldots, S_{3,37}$ do not represent pp-waves as their scalar curvatures do not vanish for any initial torsionless $B$-field. Since the actions of subgroups generated by $S_{3,14}, S_{3,28}$, $S_{3,30}, S_{3,32}, S_{3,38}$, and $S_{3,39}$ on $M$ are not transitive and free, they cannot be used for dualization.

The other subgroups provide pp-waves for suitable $B$-fields. Nevertheless, the duals obtained via $S_{3,1}, S_{3,2}, S_{3,4}, S_{3,8}, S_{3,15}, S_{3,16}, S_{3,17}, S_{3,19}, S_{3,21}, S_{3,22}, S_{3,23}, S_{3,25}, S_{3,26}$, and $S_{3,27}$ reproduce pp-waves found previously without $B$-field. Thus, we defer the discussion of these backgrounds to the Appendix with the exception of the case of $S_{3,2}$ presented below.

\subsection{Dualization with respect to subgroup generated by $S_{3,2}$}

This is rather interesting case since dualization with respect to the subgroup generated by $S_{3,2}$ produces pp-wave only for $B_{6}= \pm 1, B_{4}, B_{5}=$ const., $B_{4}^{2}-B_{5}^{2} \neq 0$. We choose $\xi(s)=\left(0, \sqrt{s_{1}}, 0,0\right)$ with $s_{1}>0$. Scalar curvature of the general dual background is

$$
\widehat{\mathcal{R}}=\frac{2\left(B_{6}^{2}-1\right)\left(-5 B_{4}^{2}+5 B_{5}^{2}+2\left(B_{6}^{2}-1\right) s_{1}\right)}{\left(B_{4}^{2}-B_{5}^{2}+\left(B_{6}^{2}-1\right) s_{1}\right)^{2}} .
$$

Setting $B_{6}= \pm 1$ gives the dual background in the form

$$
\widehat{\mathcal{F}}(s, \hat{g})=\left(\begin{array}{cccc}
\frac{1}{4 s_{1}} & 0 & 0 & 0 \\
0 & 0 & -\frac{B_{4}+B_{5} B_{6}}{B_{2}^{2}-B_{5}^{2}} & \frac{B_{5}+B_{4} B_{6}}{B_{4}^{2}-B_{5}^{2}} \\
0 & \frac{B_{4}-B_{5} B_{6}}{B_{4}^{2}-B_{5}^{2}} & \frac{B_{5}^{2}+s_{1}}{B_{4}^{2}-B_{5}^{2}} & -\frac{B_{4} B_{5}+B_{6} s_{1}}{B_{4}^{2}-B_{5}^{2}} \\
0 & \frac{B_{5}-B_{4} B_{6}}{B_{5}^{2}-B_{4}^{2}} & \frac{B_{6} s_{1}-B_{4} B_{5}}{B_{4}^{2}-B_{5}^{2}} & \frac{B_{4}^{2}-s_{1}}{B_{4}^{2}-B_{5}^{2}}
\end{array}\right) .
$$

Transformation of coordinates

$$
\begin{array}{ll}
s_{1}=z_{3}^{2}+z_{4}^{2}, & \hat{g}_{2}=-B_{5} B_{6} u+B_{4} \arctan \frac{z_{4}}{z_{3}}, \\
\hat{g}_{1}=-\frac{1}{2}\left(2 v+B_{5}^{2} u-B_{4}^{2} u\right), & \hat{g}_{3}=-B_{4} B_{6} u+B_{5} \arctan \frac{z_{4}}{z_{3}}
\end{array}
$$

eliminates the dependence on the $B$-field and we get the metric (40) and torsion (41).

\section{Duals with respect to four-dimensional subgroups}

There are no spectators in this case and the $B$-field is calculated using a constant matrix $B$, see (9). Since the actions of subgroups generated by $S_{4,3}, S_{4,4}, S_{4,5}, S_{4,9}, S_{4,10}$, 


\begin{tabular}{|c|c|c|}
\hline & Generators & Free and transitive action for \\
\hline$S_{4,1}$ & $P_{0}, P_{1}, P_{2}, P_{3}$ & $\mathbb{R}^{4}$ \\
\hline$S_{4,2}$ & $M_{3}, P_{0}-P_{3}, P_{1}, P_{2}$ & $t+z \neq 0$ \\
\hline$S_{4,3}$ & $L_{1}-M_{2}, P_{2}, P_{0}-P_{3}, L_{2}+M_{1}$ & $\emptyset$ \\
\hline$S_{4,4}$ & $L_{2}+M_{1}, P_{1}, P_{0}-P_{3}, P_{2}$ & $\emptyset$ \\
\hline$S_{4,5}$ & $\begin{array}{c}L_{1}-M_{2}-\epsilon P_{1}, P_{2}, P_{0}-P_{3}, L_{2}+M_{1}+\epsilon P_{2} \\
\epsilon= \pm 1\end{array}$ & $\emptyset$ \\
\hline$S_{4,6}$ & $L_{2}+M_{1}-\frac{1}{2}\left(P_{0}+P_{3}\right), P_{1}, P_{0}-P_{3}, P_{2}$ & $\mathbb{R}^{4}$ \\
\hline$S_{4,7}$ & $2 M_{3}+\alpha P_{1}, L_{2}+M_{1}, P_{0}-P_{3}, P_{2}, \quad \alpha>0$ & $t+z \neq 0$ \\
\hline$S_{4,8}$ & $M_{3}, L_{2}+M_{1}, P_{0}-P_{3}, P_{2}$ & $t+z \neq 0$ \\
\hline$S_{4,9}$ & $M_{3}, P_{0}, P_{3}, L_{3}$ & $\emptyset$ \\
\hline$S_{4,10}$ & $M_{3}, P_{0}, P_{3}, P_{1}$ & $\emptyset$ \\
\hline$S_{4,11}$ & $M_{3}+\alpha P_{2}, P_{0}, P_{3}, P_{1}, \quad \alpha>0$ & $\mathbb{R}^{4}$ \\
\hline$S_{4,12}$ & $L_{3}, L_{1}-M_{2}, L_{2}+M_{1}, P_{0}-P_{3}$ & $\emptyset$ \\
\hline$S_{4,13}$ & $L_{3}, P_{1}, P_{2}, M_{3}$ & $\emptyset$ \\
\hline$S_{4,14}$ & $L_{3}, P_{1}, P_{2}, P_{0}-P_{3}$ & $\emptyset$ \\
\hline$S_{4,15}$ & $L_{3}, P_{1}, P_{2}, P_{3}$ & $\emptyset$ \\
\hline$S_{4,16}$ & $L_{3}, P_{1}, P_{2}, P_{0}$ & $\emptyset$ \\
\hline$S_{4,17}$ & $L_{3}+\epsilon\left(P_{0}+P_{3}\right), P_{1}, P_{2}, P_{0}-P_{3}, \quad \epsilon= \pm 1$ & $\mathbb{R}^{4}$ \\
\hline$S_{4,18}$ & $L_{3}+\alpha P_{0}, P_{1}, P_{2}, P_{3}, \quad \alpha>0$ & $\mathbb{R}^{4}$ \\
\hline$S_{4,19}$ & $L_{3}+\alpha P_{3}, P_{1}, P_{2}, P_{0}, \quad \alpha \neq 0$ & $\mathbb{R}^{4}$ \\
\hline$S_{4,20}$ & $M_{1}, M_{2}, L_{3}, P_{3}$ & $\emptyset$ \\
\hline$S_{4,21}$ & $L_{1}, L_{2}, L_{3}, P_{0}$ & $\emptyset$ \\
\hline$S_{4,22}$ & $L_{2}+M_{1}, P_{0}+P_{3}, P_{0}-P_{3}, P_{1}$ & $\emptyset$ \\
\hline$S_{4,23}$ & $\begin{array}{c}L_{2}+M_{1}-\frac{1}{2}\left(P_{0}+P_{3}\right), L_{1}-M_{2}+\alpha P_{1}, P_{0}-P_{3}, P_{2}, \\
\alpha \neq 0\end{array}$ & $\mathbb{R}^{4}$ \\
\hline$S_{4,24}$ & $L_{2}+M_{1}-\frac{1}{2}\left(P_{0}+P_{3}\right), L_{1}-M_{2}, P_{0}-P_{3}, P_{2}$ & $\emptyset$ \\
\hline$S_{4,25}$ & $\begin{array}{c}L_{2}+M_{1}-\epsilon P_{2}, P_{0}+P_{3}, P_{1}, P_{0}-P_{3} \\
\epsilon= \pm 1\end{array}$ & $\mathbb{R}^{4}$ \\
\hline$S_{4,26}$ & $\begin{array}{c}M_{3}+\alpha P_{1}, L_{2}+M_{1}, L_{1}-M_{2}, P_{0}-P_{3} \\
\alpha>0\end{array}$ & $t+z \neq 0$ \\
\hline$S_{4,27}$ & $M_{3}, L_{1}-M_{2}, L_{2}+M_{1}, P_{0}-P_{3}$ & $t+z \neq 0$ \\
\hline$S_{4,28}$ & $\begin{array}{c}L_{3}-\tan \gamma M_{3}, L_{1}-M_{2}, L_{2}+M_{1}, P_{0}-P_{3} \\
0<\gamma<\pi, \gamma \neq \frac{\pi}{2}\end{array}$ & $t+z \neq 0$ \\
\hline$S_{4,29}$ & $\begin{array}{c}L_{3}-\tan \gamma M_{3}, P_{0}-P_{3}, P_{1}, P_{2} \\
0<\gamma<\pi, \gamma \neq \frac{\pi}{2} \\
\end{array}$ & $t+z \neq 0$ \\
\hline$S_{4,30}$ & $M_{3}, P_{1}, P_{0}-P_{3}, L_{2}+M_{1}$ & $\emptyset$ \\
\hline$S_{4,31}$ & $\begin{array}{c}M_{3}, P_{1}+\beta P_{2}, P_{0}-P_{3}, L_{2}+M_{1} \\
\beta \neq 0\end{array}$ & $t+z \neq 0$ \\
\hline$S_{4,32}$ & $\begin{array}{c}M_{3}+\alpha P_{2}, P_{1}, P_{0}-P_{3}, L_{2}+M_{1} \\
\alpha>0, \beta \neq 0\end{array}$ & $\emptyset$ \\
\hline$S_{4,33}$ & $\begin{array}{c}M_{3}+\alpha P_{2}, P_{1}+\beta P_{2}, P_{0}-P_{3}, L_{2}+M_{1} \\
\alpha>0, \beta \neq 0\end{array}$ & $t+z \neq 0$ \\
\hline$S_{4,34}$ & $\begin{array}{c}L_{3}, L_{2}+M_{1}-\epsilon P_{2}, L_{1}-M_{2}-\epsilon P_{1}, P_{0}-P_{3}, \\
\epsilon= \pm 1\end{array}$ & $\emptyset$ \\
\hline$S_{4,35}$ & $L_{3}, K_{3}, L_{2}+K_{1}, L_{1}-K_{2}$ & $\emptyset$ \\
\hline
\end{tabular}

Table 4: Generators of four-dimensional subgroups of the Poincaré group with open subsets of transitive and free action 
$S_{4,12}, \ldots, S_{4,16}, S_{4,20}, \ldots, S_{4,22}, S_{4,24}, S_{4,30}, S_{4,32}, S_{4,34}$ and $S_{4,35}$ on $M$ are not transitive and free, these subgroups cannot be used for dualization (see Tab. 44). On the other hand, for all the other subgroups the determinant $\operatorname{det}\left(E_{c d}(s)\right)$ does not vanish, and dualization is possible even with a trivial $B$-field.

Duals obtained with respect to subgroups generated by $S_{4,1}$ and $S_{4,2}$ with initial torsionless $B$-field are flat torsionless backgrounds. Dualization with respect to $S_{4,6}$ and $S_{4,11}$ gives us backgrounds where a suitable choice of constants $B_{i}$ leads to vanishing scalar curvature. However, the resulting backgrounds then become flat and torsionless.

Duals obtained with respect to subgroups generated by $S_{4,18}$ and $S_{4,19}$ do not represent pp-waves as their scalar curvatures do not vanish for any initial torsionless $B$-field.

The other subgroups provide pp-waves for suitable $B$-fields. Nevertheless, the duals obtained via $S_{4,7}, S_{4,8}, S_{4,17}, S_{4,23}, S_{4,25}, S_{4,26}, S_{4,27}, S_{4,28}, S_{4,29}, S_{4,31}$ and $S_{4,33}$ reproduce pp-waves found previously in the paper [15] without $B$-field. In other words, no new $p p$-waves are found by addition of the torsionless B-field to the flat metric. We defer the discussion of these pp-wave backgrounds to the Appendix.

\section{Conclusion}

We have investigated pp-wave backgrounds that can be found as (non-)Abelian T-duals of the flat metric supplemented by torsionless $B$-fields. It completes, together with papers [14, 15], the classification of pp-waves obtainable as (non-)Abelian T-duals of the flat Lorentzian torsionless background in four dimensions with respect to one-, two-, three-, and four-dimensional subgroups of the Poincaré group listed in [16]. Results are summarized in Tab. 5 .

There are several subgroups, namely

$$
\begin{gathered}
S_{2,3}, S_{2,7}, S_{2,9}, S_{2,18} \\
S_{3,1}, S_{3,4}, S_{3,5}, S_{3,8}, S_{3,15}, S_{3,16}, S_{3,17}, S_{3,19}, S_{3,21}, S_{3,33}, S_{3,34}
\end{gathered}
$$

that can be used for dualization only in presence of advisable non-trivial $B$-field since the condition of dualizability (14) must be satisfied. All the corresponding duals might be interpreted as complement of the classification performed in [14]. Nevertheless, we have investigated only those that produce pp-wave backgrounds.

In general considerations made in Sec. 2.2, we have identified parts of the torsionless $B$-field that influence results of the dualization, i.e., those that cannot be eliminated in principle by coordinate or gauge transformation of the dual background. This significantly simplified computations made in the following sections as we could omit several components of the initial $B$-field or set them to constants.

Plane-parallel waves can be gained by dualization with initial torsionless $B$-field with respect to subgroups generated by (see Tabs. 1,4)

$$
\begin{gathered}
S_{1,4}, S_{1,13}, \\
S_{2,2}, S_{2,3}, S_{2,8}, S_{2,12}, S_{2,13}, S_{2,21}, \\
S_{3,1}, S_{3,2}, S_{3,4}, S_{3,8}, S_{3,15}, S_{3,16}, S_{3,17}, S_{3,19}, S_{3,21}, S_{3,22}, S_{3,23}, S_{3,25}, S_{3,26}, S_{3,27},
\end{gathered}
$$




$$
S_{4,7}, S_{4,8}, S_{4,17}, S_{4,23}, S_{4,25}, S_{4,26}, S_{4,27}, S_{4,28}, S_{4,29}, S_{4,31}, S_{4,33} .
$$

All found pp-waves are of the Sfetsos-Tseytlin type, i.e., they can be expressed in the form (36) with appropriate parameters $g_{1}\left(u^{\prime}\right), g_{2}(u)$, and $q$.

It turns out that in several cases, namely for the subgroups generated by

$$
\begin{gathered}
S_{1,4}, S_{1,13}, S_{2,13}, S_{2,21}, S_{3,22}, S_{3,23}, S_{3,25}, S_{3,26}, \\
S_{4,7}, S_{4,8}, S_{4,17}, S_{4,23}, S_{4,25}, S_{4,26}, S_{4,27}, S_{4,28}, S_{4,29}, S_{4,31}, S_{4,33},
\end{gathered}
$$

the dependence of dual backgrounds on $B$-fields can be completely eliminated by coordinate and gauge transformations. It means that the addition of the $B$-field does not affect the dual model and it restores the result of [14, 15] found previously.

Dualization with respect to subgroups generated by

$$
\begin{gathered}
S_{2,3}, S_{3,1}, S_{3,17}, S_{3,21}, \\
S_{2,8}, S_{3,4}, S_{3,8}, S_{3,15}, S_{3,16}, S_{3,19}, \\
S_{3,2}, S_{3,27}
\end{gathered}
$$

reproduces pp-waves that already appeared in [14, 15] as duals with respect to some of the subgroups (44). Nevertheless, addition of suitable non-trivial $B$-field affects the dual model. Contrary to (45), subgroups generated by (46) produce different pp-wave backgrounds for different choices of initial torsionless $B$-field. Moreover, subgroups generated by (47) give pp-waves only for particular non-vanishing $B$-field.

Finally, introduction of $B$-fields yields new pp-waves obtained as T-duals of the flat torsionless background by dualization with respect to subgroups generated by

$$
S_{2,2}, S_{2,12} \text {. }
$$

They are given by the following metrics, torsions, dilatons, and the Sfetsos-Tseytlin parameters.

1. The background with

$$
\begin{gathered}
d s^{2}=\frac{2 u^{2}\left(u^{4}-5\right)}{\left(u^{4}+1\right)^{2}}\left(z_{3}^{2}+z_{4}^{2}\right) d u^{2}+2 d u d v+d z_{3}^{2}+d z_{4}^{2}, \\
\widehat{\mathcal{H}}=\frac{4 u}{u^{4}+1} d u \wedge d z_{3} \wedge d z_{4}, \\
\Phi(u)=c_{2}+c_{1} u+\log \left(u^{4}+1\right), \\
g_{1}(u)=g_{2}(u)=u^{2}, \quad q=1
\end{gathered}
$$

gained by dualization with respect to the subgroup generated by $S_{2,2}$ and initial torsionless $B$-field with $B_{6} \neq 0$.

2. One-parametric set of backgrounds with

$$
\begin{gathered}
d s_{\omega}^{2}=\left[\frac{2 u^{2}(u+1)^{4}-\omega^{2}(2 u(5 u+4)+1)}{\left(u^{2}(u+1)^{2}+\omega^{2}\right)^{2}} z_{3}^{2}+\frac{2 u^{4}(u+1)^{2}-\omega^{2}(2 u(5 u+6)+3)}{\left(u^{2}(u+1)^{2}+\omega^{2}\right)^{2}} z_{4}^{2}\right] d u^{2} \\
+2 d u d v+d z_{3}^{2}+d z_{4}^{2},
\end{gathered}
$$




$$
\begin{gathered}
\widehat{\mathcal{H}}_{\omega}=\frac{2 \omega(2 u+1)}{u^{2}(u+1)^{2}+\omega^{2}} d u \wedge d z_{3} \wedge d z_{4}, \\
\Phi_{\omega}(u)=c_{2}+c_{1} u+\log \left[u^{2}(u+1)^{2}+\omega^{2}\right], \\
g_{1}\left(u^{\prime}\right)=(1+u)^{2}, \quad g_{2}(u)=u^{2}, \quad q=\omega
\end{gathered}
$$

gained by dualization with respect to the subgroup generated by $S_{2,12}$ and initial torsionless $B$-field with $B_{6}=\omega \neq 0$.

\section{Acknowledgment}

This work was supported by the Grant Agency of the Czech Technical University in Prague, grant No. SGS16/239/OHK4/3T/14.

\section{Appendix}

Below, we summarize dual backgrounds with pp-wave metrics produced by dualization with initial torsionless $B$-fields that, however, already appeared in [14, 15] where the results were obtained without initial $B$-field. In case of one-dimensional subgroups, influence of the torsionless $B$-field on the dual backgrounds can be completely eliminated by coordinate and gauge transformations in general. Therefore, we omit these subgroups also from the Appendix and the results can be found in [14].

\section{A.1 Duals with respect to two-dimensional subgroups}

As mentioned in the beginning of Sec. 4, we can eliminate $B_{1}, \ldots, B_{5}$ and the only relevant component is the constant $B_{6}$.

\section{Dualization with respect to Abelian subgroup generated by $S_{2,8}$}

We choose $\xi(s)=\left(-\frac{s_{2}}{2 s_{1}}, 0,0, s_{1}+\frac{s_{2}}{2 s_{1}}\right)$. After elimination of $B_{1}, \ldots, B_{5}$, we get the dual background in the form

$$
\widehat{\mathcal{F}}(s, \hat{g})=\left(\begin{array}{cccc}
1-\frac{s_{2}}{s_{1}^{2}} & \frac{1}{2 s_{1}} & 0 & 0 \\
\frac{1}{2 s_{1}} & 0 & 0 & 0 \\
0 & 0 & \frac{1}{B_{6}^{2}+s_{1}^{2}} & -\frac{B_{6}}{B_{6}^{2}+s_{1}^{2}} \\
0 & 0 & \frac{B_{6}}{B_{6}^{2}+s_{1}^{2}} & \frac{s_{1}^{2}}{B_{6}^{2}+s_{1}^{2}}
\end{array}\right) .
$$

The coordinate transformation

$$
\begin{array}{ll}
s_{1}=u, & s_{2}=-u^{2}+2 u v+\frac{B_{6}^{2} z_{4}^{2}-u^{2} z_{3}^{2}}{B_{6}^{2}+u^{2}}, \\
s_{3}=z_{3} \sqrt{B_{6}^{2}+u^{2}}, & \hat{g}_{1}=\frac{z_{4} \sqrt{B_{6}^{2}+u^{2}}}{u}
\end{array}
$$




\begin{tabular}{|c|c|}
\hline Metric and torsion in Brinkmann coordinates & Generated by \\
\hline$-\left(z_{3}^{2}+z_{4}^{2}\right) d u^{2}+2 d u d v+d z_{3}^{2}+d z_{4}^{2}$ & $S_{2,3}, S_{2,13}, S_{2,21}, S_{3,2}$ \\
\hline $2 d u \wedge d z_{3} \wedge d z_{4}$ & $S_{3,27}, S_{4,17}, S_{4,29}$ \\
\hline $2 \frac{z_{3}^{2}}{u^{2}} d u^{2}+2 d u d v+d z_{3}^{2}+d z_{4}^{2}$ & $S_{1,4}, S_{2,8}, S_{3,1}$ \\
\hline 0 & $S_{3,4}, S_{3,8}, S_{3,15}$ \\
\hline $\begin{array}{c}2 \frac{z_{3}^{2}+z_{4}^{2}}{u^{2}} d u^{2}+2 d u d v+d z_{3}^{2}+d z_{4}^{2} \\
0\end{array}$ & $S_{2,2}$ \\
\hline $\begin{array}{c}2\left(\frac{z_{3}^{2}}{u^{2}}+\frac{z_{4}{ }^{2}}{(1+u)^{2}}\right) d u^{2}+2 d u d v+d z_{3}^{2}+d z_{4}^{2} \\
0\end{array}$ & $S_{2,12}$ \\
\hline $\begin{array}{c}\frac{\left(2 u^{2}-1\right) z_{3}^{2}-3 z_{4}^{2}}{\left(u^{2}+1\right)^{2}} d u^{2}+2 d u d v+d z_{3}^{2}+d z_{4}^{2} \\
\frac{2}{u^{2}+1} d u \wedge d z_{3} \wedge d z_{4}\end{array}$ & $\begin{array}{c}S_{1,13}, S_{2,8}, S_{3,4}, S_{3,8}, S_{3,15}, S_{3,16}, \\
S_{3,17}, S_{3,19}, S_{3,21}, S_{4,23}, S_{4,25}\end{array}$ \\
\hline $2 \frac{z_{3}^{2}}{\sinh ^{2} u} d u^{2}+2 d u d v+d z_{3}^{2}+d z_{4}^{2}$ & $S_{3,22}, S_{3,23}, S_{3,25}$ \\
\hline 0 & $S_{3,26}, S_{4,7}, S_{4,8}$ \\
\hline$-2 \frac{z_{3}^{2}}{\cosh ^{2} u} d u^{2}+2 d u d v+d z_{3}^{2}+d z_{4}^{2}$ & $S_{3,22}, S_{3,23}, S_{3,25}$ \\
\hline 0 & $S_{3,26}, S_{4,7}, S_{4,8}$ \\
\hline $\begin{array}{c}2 \frac{z_{3}^{2}+z_{4}^{2}}{\sinh ^{2} u} d u^{2}+2 d u d v+d z_{3}^{2}+d z_{4}^{2} \\
0\end{array}$ & $S_{4,26}, S_{4,27}$ \\
\hline $\begin{array}{c}-2 \frac{z_{3}^{2}+z_{4}^{2}}{\cosh ^{2} u} d u^{2}+2 d u d v+d z_{3}^{2}+d z_{4}^{2} \\
0\end{array}$ & $S_{4,26}, S_{4,27}$ \\
\hline $\begin{array}{c}-\left(\frac{1 \pm 2 \tan ^{2} \gamma \operatorname{sech}^{2}(u)}{\tan ^{2} \gamma}\right)\left(z_{3}^{2}+z_{4}^{2}\right) d u^{2}+2 d u d v+d z_{3}^{2}+d z_{4}^{2} \\
\frac{2}{\tan \gamma} d u \wedge d z_{3} \wedge d z_{4}\end{array}$ & $\begin{array}{c}S_{4,28} \\
0<\gamma<\pi, \gamma \neq \frac{\pi}{2}\end{array}$ \\
\hline $\begin{array}{c}-\left(\frac{4\left(2 \beta^{2} \mp\left(\beta^{2}+1\right) \cosh (2 u)+1\right)}{\left(\beta^{2} \pm\left(\beta^{2}+1\right) \cosh (2 u)-1\right)^{2}} z_{3}^{2}+\frac{4 \beta^{2}\left(\beta^{2} \pm\left(\beta^{2}+1\right) \cosh (2 u)+2\right)}{\left(\beta^{2} \pm\left(\beta^{2}+1\right) \cosh (2 u)-1\right)^{2}} z_{4}^{2}\right) d u^{2} \\
+2 d u d v+d z_{3}^{2}+d z_{4}^{2}, \\
\frac{4 \beta}{ \pm \beta^{2}+\left(\beta^{2}+1\right) \cosh (2 u) \mp 1} d u \wedge d z_{3} \wedge d z_{4}\end{array}$ & $\begin{array}{c}S_{4,31}, S_{4,33} \\
\quad \beta \neq 0\end{array}$ \\
\hline $\begin{array}{c}\frac{2 u^{2}\left(u^{4}-5\right)}{\left(u^{4}+1\right)^{2}}\left(z_{3}^{2}+z_{4}^{2}\right) d u^{2}+2 d u d v+d z_{3}^{2}+d z_{4}^{2} \\
\frac{4 u}{u^{4}+1} d u \wedge d z_{3} \wedge d z_{4}\end{array}$ & $S_{2,2}$ \\
\hline $\begin{array}{c}{\left[\frac{2 u^{2}(u+1)^{4}-\omega^{2}(2 u(5 u+4)+1)}{\left(u^{2}(u+1)^{2}+\omega^{2}\right)^{2}} z_{3}^{2}+\frac{2 u^{4}(u+1)^{2}-\omega^{2}(2 u(5 u+6)+3)}{\left(u^{2}(u+1)^{2}+\omega^{2}\right)^{2}} z_{4}^{2}\right] d u^{2}} \\
+2 d u d v+d z_{3}^{2}+d z_{4}^{2} \\
\frac{2 \omega(2 u+1)}{u^{2}(u+1)^{2}+\omega^{2}} d u \wedge d z_{3} \wedge d z_{4}\end{array}$ & $\begin{array}{l}S_{2,12} \\
\omega \neq 0\end{array}$ \\
\hline
\end{tabular}

Table 5: Complete list of pp-waves obtainable as (non-)Abelian T-duals of the flat metric with torsionless $B$-field 
brings the dual metric to the Brinkmann form

$$
d s^{2}=\frac{\left(2 u^{2}-B_{6}^{2}\right) z_{3}^{2}-3 B_{6}^{2} z_{4}^{2}}{\left(u^{2}+B_{6}^{2}\right)^{2}} d u^{2}+2 d u d v+d z_{3}^{2}+d z_{4}^{2},
$$

while the torsion reads

$$
\widehat{\mathcal{H}}=\frac{2 B_{6}}{u^{2}+B_{6}^{2}} d u \wedge d z_{3} \wedge d z_{4} .
$$

This restores the result of [14] for $B_{6}=0$. In the particular case of $B_{6} \neq 0$, the constant $B_{6}$ can be effectively rescaled to 1 by subsequent coordinate transformation, resulting in another metric and torsion that appeared already in [14, 15], see Tab. 5.

\section{Dualization with respect to Abelian subgroup generated by $S_{2,13}$}

We choose $\xi(s)=\left(0, s_{2}, s_{1}, 0\right)$. After elimination of $B_{1}, \ldots, B_{5}$, we get the dual background in the form

$$
\widehat{\mathcal{F}}(s, \hat{g})=\left(\begin{array}{cccc}
1 & 0 & 0 & -\frac{s_{2}}{B_{6}+2 \epsilon} \\
0 & 1 & 0 & \frac{s_{1}}{B_{6}+2 \epsilon} \\
0 & 0 & 0 & -\frac{1}{B_{6}+2 \epsilon} \\
-\frac{s_{2}}{B_{6}-2 \epsilon} & \frac{s_{1}}{B_{6}-2 \epsilon} & \frac{1}{B_{6}-2 \epsilon} & \frac{s_{1}^{2}+s_{2}^{2}}{B_{6}^{2}-4}
\end{array}\right)
$$

where $\epsilon= \pm 1$. Subsequent coordinate transformation

$$
\begin{array}{ll}
s_{1}=z_{3} \sin \frac{B_{6} u}{2}+z_{4} \cos \frac{B_{6} u}{2}, & \hat{g}_{1}=\epsilon s_{2}, \\
s_{2}=z_{3} \cos \frac{B_{6} u}{2}-z_{4} \sin \frac{B_{6} u}{2}, & \hat{g}_{2}=\frac{1}{2}\left(B_{6}^{2}-4\right) u
\end{array}
$$

brings the metric and torsion to the forms (40) and (41).

Dualization with respect to non-Abelian subgroup generated by $S_{2,21}$

We choose $\xi(s)=\left(\frac{1}{2}, s_{2},-s_{1}, \frac{1}{2}\right)$. After elimination of $B_{1}, \ldots, B_{5}$, the dual background acquires the form

$$
\widehat{\mathcal{F}}(s, \hat{g})=\left(\begin{array}{cccc}
1 & 0 & 0 & \frac{s_{2} \cos \gamma}{B_{6}+\left(\hat{g}_{2}-1\right) \sin \gamma} \\
0 & 1 & 0 & -\frac{s_{1} \cos \gamma}{B_{6}+\left(\hat{g}_{2}-1\right) \sin \gamma} \\
0 & 0 & 0 & \frac{1}{-B_{6}-\hat{g}_{2} \sin \gamma+\sin \gamma} \\
\frac{s_{2} \cos \gamma}{B_{6}+\left(\hat{g}_{2}+1\right) \sin \gamma} & -\frac{s_{1} \cos \gamma}{B_{6}+\left(\hat{g}_{2}+1\right) \sin \gamma} & \frac{1}{B_{6}+\left(\hat{g}_{2}+1\right) \sin \gamma} & \frac{\left(s_{1}^{2}+s_{2}^{2}\right) \cos ^{2} \gamma}{B_{6}^{2}+2 \hat{g}_{2} \sin \gamma B_{6}+\left(\hat{g}_{2}^{2}-1\right) \sin ^{2} \gamma}
\end{array}\right)
$$

where $0<\gamma<\pi, \gamma \neq \frac{\pi}{2}$. After the coordinate transformation

$$
\begin{gathered}
s_{1}=z_{3} \cos [\cot \gamma \log (\cosh (u \tan \gamma))]+z_{4} \sin [\cot \gamma \log (\cosh (u \tan \gamma))], \\
s_{2}=z_{4} \cos [\cot \gamma \log (\cosh (u \tan \gamma))]-z_{3} \sin [\cot \gamma \log (\cosh (u \tan \gamma))], \\
\hat{g}_{1}=v \cos \gamma, \quad \hat{g}_{2}=\tanh (u \tan \gamma)-\frac{1}{\sin \gamma} B_{6}
\end{gathered}
$$

valid for $\left|\hat{g}_{2}+\frac{1}{\sin \gamma} B_{6}\right|<1$, the background gives the metric (40) and torsion (41). Changing $\cosh (u \tan \gamma) \rightarrow \sinh (u \tan \gamma), \tanh (u \tan \gamma) \rightarrow \operatorname{coth}(u \tan \gamma)$ in (51), we get the transformation for $\left|\hat{g}_{2}+\frac{1}{\sin \gamma} B_{6}\right|>1$. 


\section{A.2 Duals with respect to Abelian three-dimensional subgroups}

Abelian subgroups are generated by $S_{3,1}, \ldots, S_{3,9}$. Based on the conclusions of Sec. 2.2, we find that the only relevant components of the torsionless background are

$$
B_{4}\left(s_{1}\right)=B_{4}=\text { const. }, \quad B_{5}\left(s_{1}\right)=B_{5}=\text { const. }, \quad B_{6}\left(s_{1}\right)=B_{6}=\text { const } .
$$

\section{Dualization with respect to subgroup generated by $S_{3,1}$}

This is one of the cases where dualization can be performed only for non-vanishing $B$ field, namely for $B_{5}^{2}+B_{6}^{2} \neq 0$. We choose $\xi(s)=\left(s_{1}, 0,0,0\right), s_{1} \neq 0$. After elimination of $B_{1}, B_{2}$, and $B_{3}$, we get the dual background with vanishing scalar curvature $\widehat{\mathcal{R}}$ and torsion $\widehat{\mathcal{H}}$ in the form

$$
\widehat{\mathcal{F}}(s, \hat{g})=\left(\begin{array}{cccc}
-\frac{B_{4}^{2}+u^{2}\left(B_{5}^{2}+B_{6}^{2}+u^{2}\right)}{\left(B_{5}^{2}+B_{6}^{2}\right) u^{2}} & \frac{B_{5} u^{2}+B_{4} B_{6}}{\left(B_{5}^{2}+B_{6}^{2}\right) u^{2}} & \frac{B_{6} u^{2}-B_{4} B_{5}}{\left(B_{5}^{2}+B_{6}^{2}\right) u^{2}} & \frac{u^{4}+B_{4}^{2}}{\left(B_{5}^{2}+B_{6}^{2}\right) u^{2}} \\
\frac{B_{5} u^{2}-B_{4} B_{6}}{\left(B_{5}^{2}+B_{6}^{2}\right) u^{2}} & \frac{B_{6}^{2}}{\left(B_{5}^{2}+B_{6}^{2}\right) u^{2}} & -\frac{B_{5} B_{6}}{\left(B_{5}^{2}+B_{6}^{2}\right) u^{2}} & \frac{B_{4} B_{6}-B_{5} u^{2}}{\left(B_{5}^{2}+B_{6}^{2}\right) u^{2}} \\
\frac{B_{6} u^{2}+B_{4} B_{5}}{\left(B_{5}^{2}+B_{6}^{2}\right) u^{2}} & -\frac{B_{5} B_{6}}{\left(B_{5}^{2}+B_{6}^{2}\right) u^{2}} & \frac{B_{5}^{2}}{\left(B_{5}^{2}+B_{6}^{2}\right) u^{2}} & -\frac{B_{6} u^{2}+B_{4} B_{5}}{\left(B_{5}^{2}+B_{6}^{2}\right) u^{2}} \\
-\frac{u^{4}+B_{4}^{2}}{\left(B_{5}^{2}+B_{6}^{2}\right) u^{2}} & \frac{B_{5} u^{2}+B_{4} B_{6}}{\left(B_{5}^{2}+B_{6}^{2}\right) u^{2}} & \frac{B_{6} u^{2}-B_{4} B_{5}}{\left(B_{5}^{2}+B_{6}^{2}\right) u^{2}} & \frac{u^{4}+B_{4}^{2}}{\left(B_{5}^{2}+B_{6}^{2}\right) u^{2}}
\end{array}\right) .
$$

Using the transformation of coordinates

$$
\begin{aligned}
& s_{1}=u \\
& \hat{g}_{1}=\frac{B_{5}\left(-\frac{B_{4}^{2}}{u}+B_{5}^{2} u+B_{6}^{2} u+\frac{u^{3}}{3}\right)}{2\left(B_{5}^{2}+B_{6}^{2}\right)}+\frac{B_{6}\left(x_{1}-B_{4} x_{2}\right)}{\sqrt{B_{5}^{2}+B_{6}^{2}}}+B_{5} v, \\
& \hat{g}_{2}=\frac{B_{6}\left(-\frac{B_{4}^{2}}{u}+B_{5}^{2} u+B_{6}^{2} u+\frac{u^{3}}{3}\right)}{2\left(B_{5}^{2}+B_{6}^{2}\right)}+\frac{B_{5}\left(B_{4} x_{2}-x_{1}\right)}{\sqrt{B_{5}^{2}+B_{6}^{2}}}+B_{6} v, \\
& \hat{g}_{3}=x_{2} \sqrt{B_{5}^{2}+B_{6}^{2}}
\end{aligned}
$$

the metric acquires the Rosen form 4

$$
d s^{2}=2 d u d v+\frac{1}{u^{2}} d x_{1}^{2}+u^{2} d x_{2}^{2}
$$

corresponding to the Sfetsos-Tseytlin parameters $\left(\frac{1}{u^{2}}, u^{2}, 0\right)$. The dual torsion vanishes. Consequently, it can be expressed via (32) and (33) in the Brinkmann form

$$
d s^{2}=2 \frac{z_{3}^{2}}{u^{2}} d u^{2}+2 d u d v+d z_{3}^{2}+d z_{4}^{2}
$$

that appeared already in [14].

\footnotetext{
${ }^{4}$ As mentioned in the Introduction, the Rosen form of metric is not unique in general. This metric can be also found in the Rosen form $d s^{2}=2 d u d v+\frac{1}{u^{2}} d x_{1}^{2}+d x_{2}^{2}$ corresponding to the Sfetsos-Tseytlin parameters $\left(1, u^{2}, 0\right)$.
} 


\section{Dualization with respect to subgroup generated by $S_{3,4}$}

We choose $\xi(s)=\left(s_{1}, 0,0,0\right)$ with $B_{4}^{2}+B_{6}^{2} s_{1}^{2} \neq 0, s_{1} \neq 0$. After elimination of $B_{1}, B_{2}$, and $B_{3}$, we get the dual background with vanishing scalar curvature $\widehat{\mathcal{R}}$ in the form

$$
\widehat{\mathcal{F}}(s, \hat{g})=\left(\begin{array}{cccc}
-\frac{B_{4}^{2}+B_{5}^{2}+\left(B_{6}^{2}+1\right) u^{2}}{B_{4}^{2}+B_{6}^{2} u^{2}} & \frac{B_{4}-B_{5} B_{6}}{B_{4}^{2}+B_{2}^{2} u^{2}} & \frac{B_{5}^{2}+u^{2}}{B_{4}^{2}+B_{6}^{2} u^{2}} & -\frac{B_{6} u^{2}+B_{4} B_{5}}{B_{4}^{2}+B_{6}^{2} u^{2}} \\
\frac{B_{4}+B_{5} B_{6}}{B_{4}^{2}+B_{6}^{2} u^{2}} & \frac{B_{6}^{2}}{B_{4}^{2}+B_{6}^{2} u^{2}} & -\frac{B_{4}+B_{5} B_{6}}{B_{4}^{2}+B_{6}^{2} u^{2}} & \frac{B_{4} B_{6}}{B_{4}^{2}+B_{6}^{2} u^{2}} \\
-\frac{B_{5}^{2}+u^{2}}{B_{4}^{2}+B_{6}^{2} u^{2}} & \frac{B_{4}-B_{5} B_{6}}{B_{4}^{2}+B_{6}^{2} u^{2}} & \frac{B_{5}^{2}+u^{2}}{B_{4}^{2}+B_{6}^{2} u^{2}} & -\frac{B_{6} u^{2}+B_{4} B_{5}}{B_{4}^{2}+B_{6}^{2} u^{2}} \\
\frac{B_{4} B_{5}-B_{6} u^{2}}{B_{4}^{2}+B_{6}^{2} u^{2}} & \frac{B_{4} B_{6}}{B_{4}^{2}+B_{6}^{2} u^{2}} & \frac{B_{6} u^{2}-B_{4} B_{5}}{B_{4}^{2}+B_{6}^{2} u^{2}} & \frac{B_{4}^{2}}{B_{4}^{2}+B_{6}^{2} u^{2}}
\end{array}\right) .
$$

Dual torsion vanishes for $B_{4}=0$ or $B_{6}=0$. Using the transformation of coordinates

$$
\begin{aligned}
& s_{1}=u, \\
& \hat{g}_{1}=\frac{u\left(3 B_{4}^{2}+3 B_{5}^{2}+u^{2}\right)}{6 B_{4}}+B_{4} v, \\
& \hat{g}_{2}=\frac{B_{5} B_{6} u}{B_{4}}+x_{2}, \\
& \hat{g}_{3}=\frac{B_{6}\left(-3 B_{4}^{2}(u+2 v)+3 B_{5}^{2} u+u^{3}\right)+6 B_{4} B_{5} x_{2}+6 B_{4} x_{1}}{6 B_{4}^{2}},
\end{aligned}
$$

valid for $B_{4} \neq 0$, the metric acquires the Rosen form

$$
d s^{2}=2 d u d v+\frac{1}{B_{4}^{2}+B_{6}^{2} u^{2}} d x_{1}^{2}+\frac{u^{2}}{B_{4}^{2}+B_{6}^{2} u^{2}} d x_{2}^{2} .
$$

Consequently, it can be expressed via (32) and (33) in the Brinkmann coordinates as

$$
d s^{2}=\frac{B_{6}^{2}\left(\left(2 B_{6}^{2} u^{2}-B_{4}^{2}\right) z_{3}^{2}-3 B_{4}^{2} z_{4}^{2}\right)}{\left(B_{4}^{2}+B_{6}^{2} u^{2}\right)^{2}} d u^{2}+2 d u d v+d z_{3}^{2}+d z_{4}^{2}
$$

with the corresponding torsion

$$
\widehat{\mathcal{H}}=\frac{2 B_{4} B_{6}}{B_{4}^{2}+B_{6}^{2} u^{2}} d u \wedge d z_{3} \wedge d z_{4}
$$

For $B_{6}=0$, the dual background is flat and torsionless. In the particular case of $B_{6} \neq 0$, the Brinkmann metric (53) can be transformed to the form

$$
d s^{2}=\frac{\left(2 u^{2}-B_{4}^{2}\right) z_{3}^{2}-3 B_{4}^{2} z_{4}^{2}}{\left(u^{2}+B_{4}^{2}\right)^{2}} d u^{2}+2 d u d v+d z_{3}^{2}+d z_{4}^{2}
$$

where effectively $B_{4} \in\{0,1\}$ due to additional coordinate transformation. All these backgrounds appeared already in [14, 15].

\footnotetext{
${ }^{5}$ There is a transformation for $B_{4}=0$ as well, giving the following results with $B_{4}=0$.
} 


\section{Dualization with respect to subgroup generated by $S_{3,8}$}

We choose $\xi(s)=\left(s_{1}, 0,0,0\right)$ with $B_{5}^{2}+B_{6}^{2} \neq 0, s_{1} \neq 0, s_{1} \neq-1$. After elimination of $B_{1}$, $B_{2}$, and $B_{3}$, we get the dual background with vanishing scalar curvature $\widehat{\mathcal{R}}$ in the form

$$
\begin{aligned}
& \widehat{\mathcal{F}}(s, \hat{g})= \\
& \left(\begin{array}{c}
-\frac{B_{4}^{2}+B_{5}^{2}\left(s_{1}+1\right)^{2}+s_{1}^{2}\left(B_{6}^{2}+\left(s_{1}+1\right)^{2}\right)}{B_{6}^{2} s_{1}^{2}+B_{5}^{2}\left(s_{1}+1\right)^{2}} \\
\frac{B_{5}\left(s_{1}+1\right)^{2}-B_{4} B_{6}}{B_{6}^{2} s_{1}^{2}+B_{5}^{2}\left(s_{1}+1\right)^{2}} \\
\frac{B_{6} s_{1}^{2}+B_{4} B_{5}}{B_{6}^{2} s_{1}^{2}+B_{5}^{2}\left(s_{1}+1\right)^{2}} \\
-\frac{B_{4}^{2}+s_{1}^{2}\left(s_{1}+1\right)^{2}}{B_{6}^{2} s_{1}^{2}+B_{5}^{2}\left(s_{1}+1\right)^{2}}
\end{array}\right.
\end{aligned}
$$
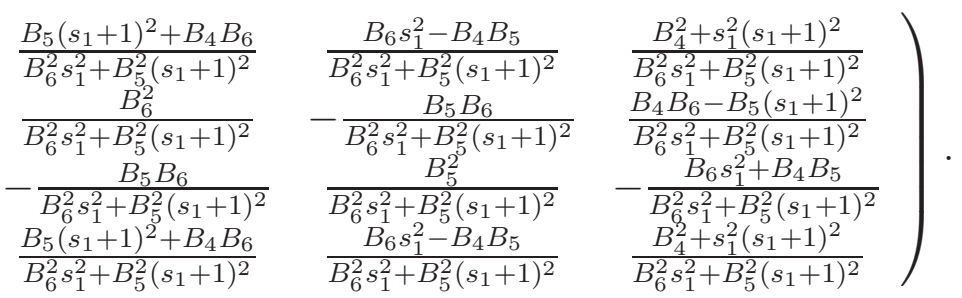

Dual torsion vanishes for $B_{5}=0$ or $B_{6}=0$. Using the transformation of coordinates

$$
\begin{aligned}
& s_{1}=u, \\
& \hat{g}_{1}=\frac{-3 B_{4}^{2}+(u+1)\left(3 B_{5}^{2}(u+2 v)+u^{3}\right)}{6 B_{5}(u+1)}, \\
& \hat{g}_{2}=\frac{B_{4}^{2} B_{6}}{2 B_{5}^{2}(u+1)}+\frac{B_{4} x_{2}}{B_{5}}-\frac{B_{6} u^{3}}{6 B_{5}^{2}}+\frac{x_{1}}{B_{5}}+\frac{B_{6} u}{2}+B_{6} v, \\
& \hat{g}_{3}=\frac{B_{4} B_{6}}{B_{5} u+B_{5}}+x_{2},
\end{aligned}
$$

valid for $6 B_{5} \neq 0$ the metric acquires the Rosen form

$$
d s^{2}=2 d u d v+\frac{1}{B_{5}^{2}(1+u)^{2}+B_{6}^{2} u^{2}} d x_{1}^{2}+\frac{u^{2}(1+u)^{2}}{B_{5}^{2}(1+u)^{2}+B_{6}^{2} u^{2}} d x_{2}^{2} .
$$

Consequently, it can be expressed via (32) and (33) in the Brinkmann coordinates as

$d s^{2}=\frac{\left(2 B_{5}^{4}(u+1)^{2}+B_{5}^{2} B_{6}^{2}\left(4 u^{2}+4 u-1\right)+2 B_{6}^{4} u^{2}\right) z_{3}^{2}-3 B_{5}^{2} B_{6}^{2} z_{4}^{2}}{\left(B_{5}^{2}(u+1)^{2}+B_{6}^{2} u^{2}\right)^{2}} d u^{2}+2 d u d v+d z_{3}^{2}+d z_{4}^{2}$

with the corresponding torsion

$$
\widehat{\mathcal{H}}=\frac{2 B_{5} B_{6}}{B_{5}^{2}(1+u)^{2}+B_{6}^{2} u^{2}} d u \wedge d z_{3} \wedge d z_{4} .
$$

For $B_{5}=0$ or $B_{6}=0$, the Brinkmann metric (54) acquires the form (52) and the torsion vanishes. In the particular case of $B_{5} \neq 0$ and $B_{6} \neq 0$, the Brinkmann metric (54) can be transformed to the form

$$
d s^{2}=\frac{\left(2 u^{2}-1\right) z_{3}^{2}-3 z_{4}^{2}}{\left(u^{2}+1\right)^{2}} d u^{2}+2 d u d v+d z_{3}^{2}+d z_{4}^{2}
$$

with torsion

$$
\widehat{\mathcal{H}}=\frac{2}{u^{2}+1} d u \wedge d z_{3} \wedge d z_{4}
$$

All these backgrounds appeared already in [14, 15].

\footnotetext{
${ }^{6}$ There is a transformation for $B_{5}=0$ as well, giving the following results with $B_{5}=0$.
} 


\section{A.3 Duals with respect to Heisenberg three-dimensional sub- groups}

Heisenberg subgroups producing pp-waves as duals of flat torsionless background are generated by $S_{3,15}, S_{3,16}, S_{3,17}, S_{3,19}$, and $S_{3,21}$. In these cases, a torsionless background on $M$ is gained if the matrix $B\left(s_{1}\right)$ fulfills

$$
B_{3}\left(s_{1}\right)=\frac{1}{K} B_{4}^{\prime}\left(s_{1}\right), \quad B_{5}\left(s_{1}\right)=B_{5}=\text { const }, \quad B_{6}\left(s_{1}\right)=B_{6}=\text { const } .
$$

where $K=\beta \neq 0$ for $S_{3,15}, K=-2 \epsilon=\mp 2$ for $S_{3,16}, S_{3,17}, K=1$ for $S_{3,19}$, and $K=-\beta \neq 0$ for $S_{3,21}$. Dependence of these dual models on $B_{1}\left(s_{1}\right), B_{2}\left(s_{1}\right), B_{3}\left(s_{1}\right)$, and $B_{4}\left(s_{1}\right)$ can be eliminated by the coordinate transformation

$$
\hat{g}_{1} \mapsto \hat{g}_{1}+\int^{s_{1}} B_{1}(r) d r, \quad \hat{g}_{2} \mapsto \hat{g}_{2}+\int^{s_{1}} B_{2}(r) d r, \quad \hat{g}_{3} \mapsto \hat{g}_{3}+\frac{1}{K} B_{4}\left(s_{1}\right)
$$

leaving the dual background dependent only on constants $B_{5}$ and $B_{6}$.

\section{Dualization with respect to subgroup generated by $S_{3,15}$}

Dualization can be performed only for $B_{5}^{2}+B_{6}^{2} \neq 0$. We choose $\xi(s)=\left(s_{1}, 0,0,0\right)$ with $\left(1+\beta^{2}\right) B_{5}^{2}+2 \beta B_{5} B_{6} s_{1}+B_{6}^{2} s_{1}^{2} \neq 0, s_{1} \neq 0$. After elimination of $B_{1}, B_{2}, B_{3}$, and $B_{4}$ by the coordinate transformation (57), the dual background $\widehat{\mathcal{F}}(s, \hat{g})$ still has rather complicated form. Nevertheless, the Ricci tensor has only one non-vanishing component

$$
\widehat{\mathcal{R}}_{s_{1} s_{1}}(s, \hat{g})=\frac{2 B_{6}^{2}\left(\left(\beta^{2}-2\right) B_{5}^{2}+2 \beta B_{5} B_{6} s_{1}+B_{6}^{2} s_{1}^{2}\right)}{\left(\left(\beta^{2}+1\right) B_{5}^{2}+2 \beta B_{5} B_{6} s_{1}+B_{6}^{2} s_{1}^{2}\right)^{2}}
$$

and scalar curvature $\widehat{\mathcal{R}}$ vanishes. It is pp-wave background with covariantly constant null vector field $k=B_{5} \partial_{\hat{g}_{1}}+B_{6} \partial_{\hat{g}_{2}}$. For $B_{6}=0$, the dual background is flat and torsionless. In the particular case of $B_{5}=0$, the dual background reads

$$
\widehat{\mathcal{F}}(s, \hat{g})=\left(\begin{array}{cccc}
-\frac{\hat{g}_{3}^{2} \beta+s_{1}^{2}}{B_{6}^{2} s_{1}^{2}}-1 & \frac{\left(s_{1}-\hat{g}_{3}\right) \beta}{B_{6} s_{1}^{2}} & \frac{1}{B_{6}} & \frac{s_{1}^{2}+\hat{g}_{3}^{2} \beta^{2}}{B_{6}^{2} s_{1}^{2}} \\
\frac{\left(\hat{g}_{3}+s_{1}\right) \beta}{B_{6} s_{1}^{2}} & \frac{1}{s_{1}^{2}} & 0 & -\frac{\left(\hat{g}_{3}+s_{1}\right) \beta}{B_{6} s_{1}^{2}} \\
\frac{1}{B_{6}} & 0 & 0 & -\frac{1}{B_{6}} \\
-\frac{s_{1}^{2}+\hat{g}_{3}^{2} \beta^{2}}{B_{6}^{2} s_{1}^{2}} & \frac{\left(s_{1}-\hat{g}_{3}\right) \beta}{B_{6} s_{1}^{2}} & \frac{1}{B_{6}} & \frac{s_{1}^{2}+\hat{g}_{3}^{2} \beta^{2}}{B_{6}^{2} s_{1}^{2}}
\end{array}\right) .
$$

Using the transformation of coordinates

$$
\begin{aligned}
& s_{1}=u, \\
& \hat{g}_{1}=-\frac{\beta u^{2}}{2 B_{6}}+\frac{1}{2} \beta B_{6} z_{4}^{2}+u z_{3}, \\
& \hat{g}_{2}=\frac{u^{2}\left(\beta^{2}+B_{6}^{2}+1\right)+B_{6}^{2}\left(-\beta^{2} z_{4}^{2}+2 u v-z_{3}^{2}\right)}{2 B_{6} u}, \\
& \hat{g}_{3}=B_{6} z_{4},
\end{aligned}
$$

the metric acquires the Brinkmann form (52) and torsion vanishes. In the case of $B_{6} \neq 0$ and $B_{5} \neq 0$, using rather complicated transformation of coordinates, the metric acquires the Brinkmann form (55) with torsion (56). 
Dualization with respect to subgroups generated by $S_{3,16}, S_{3,17}$

The algebra $S_{3,17}$ is equal to $S_{3,16}$ for $\beta=0$. Dualization with respect to $S_{3,16}, S_{3,17}$ can be performed only for non-trivial $B$-field. We choose $\xi(s)=\left(s_{1}, 0,0,0\right)$ with

$$
1+\beta s_{1}+s_{1}^{2} \neq 0, \beta^{2} B_{5}^{2}+2 \beta B_{5} B_{6} \epsilon+\left(B_{5}^{2}+B_{6}^{2}\right)+2 \beta B_{5}^{2} s_{1}+\left(B_{5}^{2}+B_{6}^{2}\right) s_{1}^{2} \neq 0 .
$$

After elimination of $B_{1}, B_{2}, B_{3}$, and $B_{4}$ by the coordinate transformation (57), the dual background $\widehat{\mathcal{F}}(s, \hat{g})$ still has rather complicated form. Nevertheless, it has simple Ricci tensor with only one non-vanishing component

$$
\begin{aligned}
& \widehat{\mathcal{R}}_{s_{1} s_{1}}(s, \hat{g})= \\
& =\frac{2\left(\beta^{2}\left(B_{5}^{4}-2 B_{5}^{2} B_{6}^{2}\right)+2 \beta B_{5}\left(B_{5}^{2}+B_{6}^{2}\right)\left(B_{5} s_{1}-2 B_{6} \epsilon\right)-\left(2-s_{1}^{2}\right)\left(B_{5}^{2}+B_{6}^{2}\right)^{2}\right)}{\left(\beta^{2} B_{5}^{2}+2 \beta B_{5}\left(B_{5} s_{1}+B_{6} \epsilon\right)+\left(s_{1}^{2}+1\right)\left(B_{5}^{2}+B_{6}^{2}\right)\right)^{2}}
\end{aligned}
$$

and vanishing scalar curvature $\widehat{\mathcal{R}}$. It is a pp-wave background with covariantly constant null vector field $k=B_{5} \partial_{\hat{g}_{1}}+B_{6} \partial_{\hat{g}_{2}}$. For $B_{5} \neq 0, B_{6} \neq 0$, and $\beta=-\varepsilon\left(\frac{B_{5}}{B_{6}}+\frac{B_{6}}{B_{5}}\right)$, using rather complicated coordinate transformation, the metric acquires the Brinkmann form (52) and the torsion vanishes. In case of $B_{5} \neq 0, B_{6} \neq 0$, and $\beta \neq-\varepsilon\left(\frac{B_{5}}{B_{6}}+\frac{B_{6}}{B_{5}}\right)$ or $B_{5}=0$ or $B_{6}=0$ or $\beta=0\left(S_{3,17}\right)$, the metric and torsion can be brought to the forms (55) and (566).

\section{Dualization with respect to subgroup generated by $S_{3,19}$}

We choose $\xi(s)=\left(s_{1}, 0,0,0\right)$ with $B_{5}^{2}+2 B_{5} B_{6} s_{1}+B_{6}^{2}\left(1+s_{1}^{2}\right) \neq 0$. After elimination of $B_{1}, B_{2}, B_{3}$, and $B_{4}$ by the coordinate transformation (57), the dual background $\widehat{\mathcal{F}}(s, \hat{g})$ still has rather complicated form, nevertheless, it has simple Ricci tensor with only one non-vanishing component

$$
\widehat{\mathcal{R}}_{s_{1} s_{1}}(s, \hat{g})=\frac{2 B_{6}^{2}\left(B_{5}^{2}+2 B_{5} B_{6} s_{1}+B_{6}^{2}\left(-2+s_{1}^{2}\right)\right)}{B_{5}^{2}+2 B_{5} B_{6} s_{1}+B_{6}^{2}\left(1+s_{1}^{2}\right)}
$$

and vanishing scalar curvature $\widehat{\mathcal{R}}$. It is pp-wave background with covariantly constant null vector field $k=B_{5} \partial_{\hat{g}_{1}}+B_{6} \partial_{\hat{g}_{2}}$. For $B_{6}=0$, the dual background is flat and torsionless. In the particular case of $B_{5}=0$, the metric can be brought to the Brinkmann form (52) and torsion vanishes. In the particular case of $B_{5} \neq 0$ and $B_{6} \neq 0$, the dual metric acquires the Brinkmann form (55) with torsion (56) after the coordinate transformation

$$
\begin{aligned}
s_{1}= & u-\frac{B_{5}}{B_{6}}, \\
\hat{g}_{1}= & \frac{B_{5}\left(u^{3}\left(1-B_{6}^{2}\left(z_{4}^{2}-1\right)\right)+2 B_{6}^{2} u^{2} v+u\left(1-B_{6}^{2}\left(z_{3}^{2}+2 z_{4}^{2}-1\right)\right)+2 B_{6}^{2} v\right)}{2 B_{6}^{2}\left(u^{2}+1\right)}+ \\
& \frac{u^{2}\left(B_{6}^{2} z_{4}^{2}-1\right)+B_{6}^{2} z_{4}^{2}}{2 B_{6}}+\sqrt{1+u^{2}} z_{3}, \\
\hat{g}_{2}= & \frac{u^{3}\left(1-B_{6}^{2}\left(z_{4}^{2}-1\right)\right)+2 B_{6}^{2} u^{2} v+u\left(1-B_{6}^{2}\left(z_{3}^{2}+2 z_{4}^{2}-1\right)\right)+2 B_{6}^{2} v}{2 B_{6}\left(u^{2}+1\right)}, \\
\hat{g}_{3}= & B_{6} \sqrt{1+u^{2}} z_{4} .
\end{aligned}
$$




\section{Dualization with respect to subgroup generated by $S_{3,21}$}

We choose $\xi(s)=\left(s_{1}, 0,0,0\right)$ with $\left(1+\beta^{2}\right) B_{5}^{2}+2 B_{5} B_{6} \epsilon+B_{6}^{2} \epsilon^{2}-2 \beta B_{5} B_{6} s_{1}+B_{6}^{2} s_{1}^{2} \neq 0$, $s_{1}+\beta \epsilon \neq 0$. After elimination of $B_{1}, B_{2}, B_{3}$, and $B_{4}$ by the coordinate transformation (57), the dual background $\widehat{\mathcal{F}}(s, \hat{g})$ still has rather complicated form, nevertheless, it has simple Ricci tensor with only one non-vanishing component

$$
\widehat{\mathcal{R}}_{s_{1} s_{1}}(s, \hat{g})=\frac{2 B_{6}^{2}\left(\left(\beta^{2}-2\right) B_{5}^{2}-2 \beta B_{5} B_{6} s_{1}-4 B_{5} B_{6} \epsilon-2 B_{6}^{2} \epsilon^{2}+B_{6}^{2} s_{1}^{2}\right)}{\left(\left(\beta^{2}+1\right) B_{5}^{2}-2 \beta B_{5} B_{6} s_{1}+2 B_{5} B_{6} \epsilon+B_{6}^{2} \epsilon^{2}+B_{6}^{2} s_{1}^{2}\right)^{2}}
$$

and vanishing scalar curvature $\widehat{\mathcal{R}}$. It is pp-wave background with covariantly constant null vector field $k=B_{5} \partial_{\hat{g}_{1}}+B_{6} \partial_{\hat{g}_{2}}$. For $B_{6}=0$, the dual background is flat and torsionless. In the particular case of $B_{6} \neq 0$, the metric and torsion can be brought to the forms (55) and (56).

\section{A.4 Duals with respect to subgroups generated by Bianchi 4, Bianchi 5}

Dualization with respect to subgroups generated by $S_{3,22}, S_{3,25}$

In these cases, a torsionless background on $M$ is obtained if the matrix $B\left(s_{1}\right)$ fulfills

$$
B_{2}\left(s_{1}\right)=-B_{4}^{\prime}\left(s_{1}\right)+\alpha B_{5}^{\prime}\left(s_{1}\right), \quad B_{3}\left(s_{1}\right)=-B_{5}^{\prime}\left(s_{1}\right), \quad B_{6}\left(s_{1}\right)=0
$$

where $\alpha=0$ for $S_{3,25}$ and $\alpha>0$ for $S_{3,22}$. We choose $\xi(s)=\left(\frac{1}{2}, 0, s_{1}, \frac{1}{2}\right)$. The sigma model is dualizable for $B_{5}\left(s_{1}\right) \neq \pm 1$. The dual background $\widehat{\mathcal{F}}(s, \hat{g})$ has rather complicated form with vanishing scalar curvature $\widehat{\mathcal{R}}$ and torsion $\widehat{\mathcal{H}}$. Dependence of the dual model on components of the matrix $B\left(s_{1}\right)$ can be eliminated by the coordinate transformation

$$
\hat{g}_{1} \mapsto \hat{g}_{1}+\int^{s_{1}} B_{1}(r) d r, \quad \hat{g}_{2} \mapsto \hat{g}_{2}-B_{4}\left(s_{1}\right)+\alpha B_{5}\left(s_{1}\right), \quad \hat{g}_{3} \mapsto \hat{g}_{3}-B_{5}\left(s_{1}\right)
$$

that brings the dual background to the form

$$
\widehat{\mathcal{F}}(s, \hat{g})=\left(\begin{array}{cccc}
1 & 0 & 0 & 0 \\
0 & 0 & 0 & \frac{1}{1-\hat{g}_{3}} \\
0 & 0 & 1 & \frac{\hat{g}_{3}++\alpha+\hat{g}_{2}}{1-\hat{g}_{3}} \\
0 & \frac{1}{\hat{g}_{3}+1} & \frac{-\hat{g}_{3} \alpha+\alpha-\hat{g}_{2}}{\hat{g}_{3}+1} & \frac{\left(\hat{g}_{2}+\alpha \hat{g}_{3}\right)^{2}}{\hat{g}_{3}^{2}-1}
\end{array}\right) .
$$

Using the coordinate transformation

$$
\begin{aligned}
& s_{1}=u, \\
& \hat{g}_{1}=\frac{\alpha^{2}}{2} u+v+\alpha z_{3}(\log (\cosh u)-1)-\frac{1}{2} \tanh u\left[\alpha^{2}\left((\log (\cosh u)-1)^{2}+z_{3}^{2}\right)\right] \\
& \hat{g}_{2}=z_{3}-\alpha \tanh u \log (\cosh u), \\
& \hat{g}_{3}=\tanh u
\end{aligned}
$$


for $\left|\hat{g}_{3}\right|<1$, the dual metric acquires the Brinkmann form

$$
d s^{2}=-2 \frac{z_{3}^{2}}{\cosh ^{2} u} d u^{2}+2 d u d v+d z_{3}^{2}+d z_{4}^{2}
$$

Changing $\cosh u \rightarrow \sinh u$ and $\tanh u \rightarrow \operatorname{coth} u$ in the transformation, we get

$$
d s^{2}=2 \frac{z_{3}^{2}}{\sinh ^{2} u} d u^{2}+2 d u d v+d z_{3}^{2}+d z_{4}^{2}
$$

for $\left|\hat{g}_{3}\right|>1$. Torsion in both cases vanishes. This restores the result of [14] for any initial torsionless $B$-field. The background appeared already in [14, 15] by dualization with respect to subgroups generated by $S_{3,22}, S_{3,23}, S_{3,25}, S_{3,26}, S_{4,7}$, and $S_{4,8}$ without $B$-field.

\section{Dualization with respect to subgroups generated by $S_{3,23}, S_{3,26}$}

In these cases, a torsionless background on $M$ is obtained if the matrix $B\left(s_{1}\right)$ fulfills

$$
B_{2}\left(s_{1}\right)=-B_{4}^{\prime}\left(s_{1}\right)-\beta B_{3}\left(s_{1}\right), \quad B_{3}\left(s_{1}\right)=-B_{5}^{\prime}\left(s_{1}\right), \quad B_{6}\left(s_{1}\right)=0
$$

where $\beta=0$ for $S_{3,26}$ and $\beta \neq 0$ for $S_{3,23}$. We choose $\xi(s)=\left(\frac{\sigma}{2}, 0, \alpha \log \left|s_{1}\right|, \frac{\sigma}{2}\right)$, $\sigma=\operatorname{sgn}\left(s_{1}\right), s_{1} \neq 0$. The sigma model is dualizable for $B_{5}\left(s_{1}\right) \neq \pm 1$. The dual background $\widehat{\mathcal{F}}(s, \hat{g})$ has rather complicated form with vanishing scalar curvature $\widehat{\mathcal{R}}$ and torsion $\widehat{\mathcal{H}}$. Dependence of the dual model on components of the matrix $B\left(s_{1}\right)$ can be eliminated by the coordinate transformation

$$
\hat{g}_{1} \mapsto \hat{g}_{1}+\int^{s_{1}} B_{1}(r) d r, \quad \hat{g}_{2} \mapsto \hat{g}_{2}-B_{4}\left(s_{1}\right)+\beta B_{5}\left(s_{1}\right), \quad \hat{g}_{3} \mapsto \hat{g}_{3}-B_{5}\left(s_{1}\right)
$$

that brings the dual background to the form

$$
\widehat{\mathcal{F}}(s, \hat{g})=\left(\begin{array}{cccc}
\frac{\alpha^{2}}{s_{1}^{2}} & 0 & 0 & -\frac{\alpha^{2}}{s_{1}\left(\sigma-\hat{g}_{3}\right)} \\
0 & 0 & 0 & \frac{1}{\sigma-\hat{g}_{3}} \\
0 & 0 & 1 & \frac{\beta \sigma+\hat{g}_{2}+\beta \hat{g}_{3}}{\sigma-\hat{g}_{3}} \\
\frac{\alpha^{2}}{s_{1}\left(\sigma+\hat{g}_{3}\right)} & \frac{1}{\sigma+\hat{g}_{3}} & -\frac{-\beta \sigma+\hat{g}_{2}+\beta \hat{g}_{3}}{\sigma+\hat{g}_{3}} & -\frac{\alpha^{2}+\left(\hat{g}_{2}+\beta \hat{g}_{3}\right)^{2}}{1-\hat{g}_{3}^{2}}
\end{array}\right)
$$

where $\alpha>0$ for both subgroups. By rather complicated coordinate transformation (see [14]), the dual metric acquires the Brinkmann form (58) or (59). Torsion in both cases vanishes.

\section{A.5 Dual with respect to subgroup generated by Bianchi $6_{0}$}

Dualization with respect to subgroup generated by $S_{3,27}$

In this case, a torsionless background on $M$ is obtained if the matrix $B\left(s_{1}\right)$ fulfills

$$
B_{2}\left(s_{1}\right)=\csc \gamma B_{5}^{\prime}\left(s_{1}\right), \quad B_{3}\left(s_{1}\right)=\csc \gamma B_{4}^{\prime}\left(s_{1}\right), \quad B_{6}\left(s_{1}\right)=B_{6}=\text { const } .
$$

where $0<\gamma<\pi, \gamma \neq \frac{\pi}{2}$. We choose $\xi(s)=\left(0,0, \sqrt{s_{1}}, 0\right)$ with $B_{4}\left(s_{1}\right)^{2}-B_{5}\left(s_{1}\right)^{2}+\left(B_{6}^{2}-\right.$ 1) $s_{1} \cos ^{2} \gamma \neq 0, s_{1}>0$. Dual scalar curvature $\widehat{\mathcal{R}}$ vanishes for $B_{6}= \pm 1$ and we get pp-wave 
background with covariantly constant null vector field $\partial_{\hat{g}_{1}}$. Using the transformation of coordinates

$$
\begin{aligned}
& s_{1}=z_{3}^{2}+z_{4}^{2}, \\
& \hat{g}_{1}=-B_{6} v \cos \gamma-\frac{\sin \gamma}{4} e^{2 u \tan \gamma}+\int^{z_{3}^{2}+z_{4}^{2}} B_{1}(r) d r, \\
& \hat{g}_{2}=e^{u \tan \gamma} \cosh \left(\tan (\gamma) \arctan \frac{z_{4}}{z_{3}}\right)+\csc \gamma B_{5}\left(z_{3}^{2}+z_{4}^{2}\right), \\
& \hat{g}_{3}=-e^{u \tan \gamma} \sinh \left(\tan (\gamma) \arctan \frac{z_{4}}{z_{3}}\right)+\csc \gamma B_{4}\left(z_{3}^{2}+z_{4}^{2}\right),
\end{aligned}
$$

the dual background with $B_{6}= \pm 1$ acquires the Brinkmann form77 (40) with torsion (41).

\section{A.6 Duals with respect to four-dimensional subgroups}

For four-dimensional subgroups the matrix $B$ is constant. Since the conditions on the initial $B$-field

$$
\mathcal{H}=d \mathcal{B}=0
$$

differ case by case, we shall give the solutions when discussing particular dual backgrounds.

\section{Dualization with respect to subgroups generated by $S_{4,7}, S_{4,8}$}

As we can see from Tab. 4, non-isomorphic subalgebras $S_{4,7}$ and $S_{4,8}$ differ in the value of the parameter $\alpha$, which is positive for $S_{4,7}$ and vanishes for $S_{4,8}$. We choose $\xi(s)=$ $\left(\frac{1}{2}, 0,0, \frac{1}{2}\right)$. In both cases the conditions (60) hold when $B_{4}=B_{5}=B_{6}=0$. The scalar curvatures $\widehat{\mathcal{R}}$ as well as torsions $\widehat{\mathcal{H}}$ then vanish. The symmetric parts of both backgrounds $\widehat{\mathcal{F}}$ can be brought to Brinkmann forms (58) or (59) that are independent of the parameter $\alpha$. As the formulas for $S_{4,7}$ are too complicated to display, we give only the results valid for $S_{4,8}$.

The symmetric part of dual background

$$
\widehat{\mathcal{F}}\left(\hat{g}_{1}, \hat{g}_{2}, \hat{g}_{3}, \hat{g}_{4}\right)=\left(\begin{array}{cccc}
0 & 0 & -\frac{1}{B_{2}+\hat{g}_{3}-1} & 0 \\
0 & 1 & -\frac{B_{1}+\hat{g}_{2}}{B_{2}+\hat{g}_{3}-1} & 0 \\
\frac{1}{B_{2}+\hat{g}_{3}+1} & -\frac{B_{1}+\hat{g}_{2}}{B_{2}+\hat{g}_{3}+1} & \frac{B_{1}^{2}+2 \hat{g}_{2} B_{1}+B_{3}^{2}+\hat{g}_{2}^{2}}{B_{2}^{2}+2 \hat{g}_{3} B_{2}+\hat{g}_{3}^{2}-1} & -\frac{B_{3}}{B_{2}+\hat{g}_{3}+1} \\
0 & 0 & -\frac{B_{3}}{B_{2}+\hat{g}_{3}-1} & 1
\end{array}\right)
$$

can be brought to Brinkmann form (58) using transformation

$$
\begin{array}{ll}
\hat{g}_{1}=v+\frac{1}{2} B_{3}^{2} u-\frac{1}{2} z_{3}^{2} \tanh (u), & \hat{g}_{3}=\tanh (u)-B_{2}, \\
\hat{g}_{2}=z_{3}-B_{1}, & \hat{g}_{4}=z_{4}-B_{3} \log (\cosh (u))
\end{array}
$$

for $\left|\hat{g}_{3}+B_{2}\right|<1$, or to the form (59) for $\left|\hat{g}_{3}+B_{2}\right|>1$ using similar transformation where we change tanh $\rightarrow$ coth and cosh $\rightarrow$ sinh.

\footnotetext{
${ }^{7}$ For $B_{6}=-1$, it is necessary to interchange cosh and sinh.
} 


\section{Dualization with respect to subgroup generated by $S_{4,17}$}

We choose $\xi(s)=(0,0,0,0)$. When conditions (60) hold, i.e. when $B_{5}=B_{6}=0$, the shift

$$
\hat{g}_{2} \mapsto \hat{g}_{2}-B_{2}, \quad \hat{g}_{3} \mapsto \hat{g}_{3}+B_{1}
$$

simplifies the dual background to the form

$$
\widehat{\mathcal{F}}\left(\hat{g}_{1}, \hat{g}_{2}, \hat{g}_{3}, \hat{g}_{4}\right)=\left(\begin{array}{cccc}
0 & 0 & 0 & -\frac{1}{2 \epsilon+B_{3}} \\
0 & \frac{1}{B_{4}^{2}+1} & -\frac{B_{4}}{B_{4}^{2}+1} & \frac{B_{4} \hat{g}_{2}+\hat{g}_{3}}{\left(2 \epsilon+B_{3}\right)\left(B_{4}^{2}+1\right)} \\
0 & \frac{B_{4}}{B_{4}^{2}+1} & \frac{1}{B_{4}^{2}+1} & \frac{B_{4} \hat{g}_{3}-\hat{g}_{2}}{\left(2 \epsilon+B_{3}\right)\left(B_{4}^{2}+1\right)} \\
\frac{1}{B_{3}-2 \epsilon} & \frac{B_{4} \hat{g}_{2}-\hat{g}_{3}}{\left(2 \epsilon-B_{3}\right)\left(B_{4}^{2}+1\right)} & \frac{\hat{g}_{2}+B_{4} \hat{g}_{3}}{\left(2 \epsilon-B_{3}\right)\left(B_{4}^{2}+1\right)} & -\frac{\hat{g}_{2}^{2}+\hat{g}_{3}^{2}}{\left(4 \epsilon^{2}-B_{3}^{2}\right)\left(B_{4}^{2}+1\right)}
\end{array}\right)
$$

where $\epsilon= \pm 1$. Subsequent coordinate transformation

$$
\begin{array}{ll}
\hat{g}_{1}=v+\frac{B_{4}}{2}\left(z_{3}^{2}+z_{4}^{2}\right), & \hat{g}_{3}=\sqrt{B_{4}^{2}+1}\left(z_{3} \sin \frac{B_{3} u}{2 \epsilon}+z_{4} \cos \frac{B_{3} u}{2 \epsilon}\right), \\
\hat{g}_{4}=u\left(\frac{B_{3}^{2}}{2 \epsilon}-2 \epsilon\right), & \hat{g}_{2}=\sqrt{B_{4}^{2}+1}\left(z_{3} \cos \frac{B_{3} u}{2 \epsilon}-z_{4} \sin \frac{B_{3} u}{2 \epsilon}\right)
\end{array}
$$

brings the metric to Brinkmann form (40). The corresponding torsion is (41).

\section{Dualization with respect to subgroup generated by $S_{4,23}$}

We choose $\xi(s)=(0,0,0,0)$. Conditions (60) hold when $B_{2}=B_{6}=0$. After the shift

$$
\hat{g}_{3} \mapsto \hat{g}_{3}-B_{5}, \quad \hat{g}_{4} \mapsto \hat{g}_{4}-B_{1}
$$

the dual background is still too complicated to display, but its scalar curvature $\widehat{\mathcal{R}}$ is given by

$$
\widehat{\mathcal{R}}=\frac{14\left(B_{4}^{4}+B_{4}^{2}\right)}{\left(2 \alpha B_{4} \hat{g}_{3}+2 B_{5} \hat{g}_{3}-2 B_{4} \hat{g}_{4}+\hat{g}_{3}^{2}+\alpha^{2}-B_{3}^{2} B_{4}^{2}+B_{5}^{2}-2 B_{1} B_{4}\right)^{2}},
$$

where $\alpha \neq 0$. Scalar curvature vanishes for $B_{4}=0$, in which case the dual background reads

$$
\begin{aligned}
& \widehat{\mathcal{F}}\left(\hat{g}_{1}, \hat{g}_{2}, \hat{g}_{3}, \hat{g}_{4}\right)= \\
& \left(\begin{array}{cccc}
0 & 0 & 1 & 0 \\
0 & \frac{1}{\alpha^{2}+\hat{g}_{3}^{2}} & \frac{\alpha B_{5}-\alpha \hat{g}_{3}-B_{3} \hat{g}_{3}+\hat{g}_{4}}{\alpha^{2}+\hat{g}_{3}^{2}} & -\frac{\hat{g}_{3}}{\alpha^{2}+\hat{g}_{3}^{2}} \\
1 & -\frac{\alpha B_{5}-\alpha \hat{g}_{3}+B_{3} \hat{g}_{3}+\hat{g}_{4}}{\alpha^{2}+\hat{g}_{3}^{2}} & -\frac{\left(B_{3}^{2}+\left(B_{5}-\hat{g}_{3}\right)^{2}\right) \alpha^{2}+2\left(B_{5}-\hat{g}_{3}\right) \hat{g}_{4} \alpha+\hat{g}_{4}^{2}}{\alpha^{2}+\hat{g}_{3}^{2}} & \frac{\left(B_{5}-\hat{g}_{3}\right) \hat{g}_{3} \alpha-\alpha^{2} B_{3}+\hat{g}_{3} \hat{g}_{4}}{\alpha^{2}+\hat{g}_{3}^{2}} \\
0 & \frac{\hat{g}_{3}}{\alpha^{2}+\hat{g}_{3}^{2}} & \frac{B_{3} \alpha^{2}+\left(B_{5}-\hat{g}_{3} \hat{g}_{3} \alpha+\hat{g}_{3} \hat{g}_{4}\right.}{\alpha^{2}+\hat{g}_{3}^{2}} & \frac{\alpha^{2}}{\alpha^{2}+\hat{g}_{3}^{2}}
\end{array}\right) .
\end{aligned}
$$


Subsequent coordinate transformation

$$
\begin{aligned}
\hat{g}_{1}= & -\frac{v}{\alpha}+\frac{-12 \alpha^{2} B_{3}^{2} u+\alpha^{4} u\left(3 u^{4}-28 u^{2}+48\right)}{24 \alpha} \\
& +2 \alpha \sqrt{u^{2}+1} z_{2}-\frac{1}{2} \alpha u^{2} \sqrt{u^{2}+1} z_{2}+\frac{u\left(\left(u^{2}+2\right) z_{2}^{2}+z_{3}^{2}\right)}{2 \alpha\left(u^{2}+1\right)}, \\
\hat{g}_{2}= & \frac{1}{2} \alpha\left(\alpha B_{3} u^{2}+2 \sqrt{u^{2}+1} z_{3}\right), \\
\hat{g}_{3}= & -\alpha u \\
\hat{g}_{4}= & -\alpha B_{5}-\frac{1}{2} \alpha^{2} u\left(u^{2}-4\right)+\sqrt{u^{2}+1} z_{2}
\end{aligned}
$$

brings the metric to Brinkmann form (55). The corresponding torsion is (56).

\section{Dualization with respect to subgroup generated by $S_{4,25}$}

We choose $\xi(s)=(0,0,0,0)$. Conditions (60) hold when $B_{5}=B_{6}=0$. After the shift

$$
\hat{g}_{3} \mapsto \hat{g}_{3}+\frac{B_{1}}{2}, \quad \hat{g}_{4} \mapsto \hat{g}_{4}+B_{2}
$$

the dual background is still too complicated to display, but its scalar curvature $\widehat{\mathcal{R}}$ is given by

$$
\widehat{\mathcal{R}}=\frac{224 B_{3}^{2}}{\left(8 B_{3} \hat{g}_{3}+4 \hat{g}_{4}^{2}-B_{3}^{2} B_{4}^{2}+4 \epsilon^{2}\right)^{2}},
$$

where $\epsilon= \pm 1$. Scalar curvature vanishes for $B_{3}=0$, in which case the dual background reads

$$
\widehat{\mathcal{F}}\left(\hat{g}_{1}, \hat{g}_{2}, \hat{g}_{3}, \hat{g}_{4}\right)=\left(\begin{array}{cccc}
\frac{1}{\epsilon^{2}+\hat{g}_{4}^{2}} & 0 & \frac{\hat{g}_{4}}{\epsilon^{2}+\hat{g}_{4}^{2}} & -\frac{2 \hat{g}_{3}+B_{4} \hat{g}_{4}}{2\left(\epsilon^{2}+\hat{g}_{4}^{2}\right)} \\
0 & 0 & 0 & -\frac{1}{2} \\
-\frac{\hat{g}_{4}}{\epsilon^{2}+\hat{g}_{4}^{2}} & 0 & \frac{\epsilon^{2}}{\epsilon^{2}+\hat{g}_{4}^{2}} & \frac{2 \hat{g}_{3} \hat{g}_{4}-\epsilon^{2} B_{4}}{2\left(\epsilon^{2}+\hat{g}_{4}^{2}\right)} \\
\frac{2 \hat{g}_{3}-B_{4} \hat{g}_{4}}{2\left(\epsilon^{2}+\hat{g}_{4}^{2}\right)} & -\frac{1}{2} & \frac{B_{4} \epsilon^{2}+2 \hat{g}_{3} \hat{g}_{4}}{2\left(\epsilon^{2}+\hat{g}_{4}^{2}\right)} & -\frac{\epsilon^{2} B_{4}^{2}+4 \hat{g}_{3}^{2}}{4\left(\epsilon^{2}+\hat{g}_{4}^{2}\right)}
\end{array}\right) \text {. }
$$

Subsequent coordinate transformation

$$
\begin{array}{ll}
\hat{g}_{1}=\frac{B_{4} u^{2} \epsilon^{2}}{4}+\epsilon \sqrt{u^{2}+1} z_{3}, & \hat{g}_{3}=\sqrt{u^{2}+1} z_{4}, \\
\hat{g}_{2}=-\frac{2 v}{\epsilon}+\frac{u\left(4\left(\left(u^{2}+2\right) z_{4}^{2}+z_{3}^{2}\right)-B_{4}^{2}\left(u^{2}+1\right) \epsilon^{2}\right)}{4\left(u^{2}+1\right) \epsilon}, & \hat{g}_{4}=u \epsilon
\end{array}
$$

brings the metric to Brinkmann form (55). The corresponding torsion is (56).

\section{Dualization with respect to subgroups generated by $S_{4,26}, S_{4,27}$}

As we can see from Tab. 4, non-isomorphic subalgebras $S_{4,26}$ and $S_{4,27}$ differ only in the value of the parameter $\alpha$. Accordingly, the results of dualization and corresponding coordinate transformations can be written concisely in terms of $\alpha$, which is positive for $S_{4,26}$ and vanishes for $S_{4,27}$. We choose $\xi(s)=\left(\frac{1}{2}, 0,0, \frac{1}{2}\right)$. For both subalgebras the 
conditions (60) hold when $B_{4}=B_{5}=B_{6}=0$. The scalar curvatures $\widehat{\mathcal{R}}$ as well as torsions $\widehat{\mathcal{H}}$ then vanish. The shift

$$
\hat{g}_{2} \mapsto \hat{g}_{2}-B_{1}, \quad \hat{g}_{3} \mapsto \hat{g}_{3}-B_{2}, \quad \hat{g}_{4} \mapsto \hat{g}_{4}-B_{3}
$$

simplifies the dual background to the form

$$
\widehat{\mathcal{F}}\left(\hat{g}_{1}, \hat{g}_{2}, \hat{g}_{3}, \hat{g}_{4}\right)=\left(\begin{array}{cccc}
0 & 0 & 0 & \frac{1}{1-\hat{g}_{4}} \\
0 & 1 & 0 & \frac{-B_{3} \alpha+\hat{g}_{g} \alpha+\alpha+\hat{g}_{2}}{1-\hat{g}_{4}} \\
0 & 0 & 1 & -\frac{\hat{g}_{3}}{\hat{g}_{4}-1} \\
\frac{1}{\hat{g}_{4}+1} & \frac{B_{3} \alpha-\hat{g}_{4} \alpha+\alpha-\hat{g}_{2}}{\hat{g}_{4}+1} & -\frac{\hat{g}_{3}}{\hat{g}_{4}+1} & \frac{\hat{g}_{2}^{2}+2 \alpha\left(\hat{g}_{4}-B_{3}\right) \hat{g}_{2}+\hat{g}_{3}^{2}+\alpha^{2}\left(B_{3}-\hat{g}_{4}\right)^{2}}{\hat{g}_{4}^{2}-1}
\end{array}\right) \text {. }
$$

Subsequent coordinate transformation

$$
\begin{aligned}
\hat{g}_{1}= & v+\frac{1}{2}\left(-\alpha z_{3}\left(2 B_{3} u+\log (1-\tanh (u))+\log (\tanh (u)+1)+2\right)\right) \\
& -\frac{1}{8} \alpha^{2} \tanh (u)\left(2 B_{3} u+\log (1-\tanh (u))+\log (\tanh (u)+1)+2\right)^{2} \\
& +\frac{1}{2} \alpha^{2}\left(B_{3}^{2}+1\right) u-\alpha^{2} B_{3} \log (\cosh (u))-\frac{1}{2}\left(z_{3}^{2}+z_{4}^{2}\right) \tanh (u), \\
\hat{g}_{2}= & z_{3}+\frac{1}{2} \alpha \tanh (u)\left(2 B_{3} u+\log (1-\tanh (u))+\log (\tanh (u)+1)\right), \\
\hat{g}_{3}= & z_{4}, \\
\hat{g}_{4}= & \tanh (u)
\end{aligned}
$$

valid for $\left|\hat{g}_{4}\right|<1$ brings the metric to $\alpha$-independent Brinkmann form

$$
d s^{2}=-2 \frac{z_{3}^{2}+z_{4}^{2}}{\cosh ^{2} u} d u^{2}+2 d u d v+d z_{3}^{2}+d z_{4}^{2} .
$$

For $\left|\hat{g}_{4}\right|>1$ similar transformation with tanh $\rightarrow$ coth and cosh $\rightarrow$ sinh gives metric

$$
d s^{2}=2 \frac{z_{3}^{2}+z_{4}^{2}}{\sinh ^{2} u} d u^{2}+2 d u d v+d z_{3}^{2}+d z_{4}^{2} .
$$

This restores the results of [15].

\section{Dualization with respect to subgroup generated by $S_{4,28}$}

We choose $\xi(s)=\left(\frac{1}{2}, 0,0, \frac{1}{2}\right)$. When the conditions (60) hold, i.e. when $B_{4}=B_{5}=B_{6}=$ 0 , the scalar curvature $\widehat{\mathcal{R}}$ vanishes. We denote $\beta:=\tan (\gamma)$, where $0<\gamma<\pi, \gamma \neq \frac{\pi}{2}$, see Tab. 4. The shift

$$
\hat{g}_{4} \mapsto \hat{g}_{4}+\frac{B_{3}}{\beta}
$$

simplifies the dual background to the form

$$
\begin{aligned}
& \widehat{\mathcal{F}}\left(\hat{g}_{1}, \hat{g}_{2}, \hat{g}_{3}, \hat{g}_{4}\right)= \\
& \left(\begin{array}{cccc}
0 & 0 & 0 & \frac{1}{\beta\left(\hat{g}_{4}-1\right)} \\
0 & 1 & 0 & \frac{B_{1}-\beta \hat{g}_{2}+\hat{g}_{3}}{\beta\left(\hat{g}_{4}-1\right)} \\
0 & 0 & 1 & \frac{B_{2}-\hat{g}_{2}-\beta \hat{g}_{3}}{\beta\left(\hat{g}_{4}-1\right)} \\
-\frac{1}{\hat{g}_{4} \beta+\beta} & \frac{B_{1}-\beta \hat{g}_{2}+\hat{g}_{3}}{\hat{g}_{4} \beta+\beta} & \frac{B_{2}-\hat{g}_{2}-\beta \hat{g}_{3}}{\hat{g}_{4} \beta+\beta} & \frac{B_{1}^{2}+2\left(\hat{g}_{3}-\beta \hat{g}_{2}\right) B_{1}+B_{2}^{2}-2 B_{2}\left(\hat{g}_{2}+\beta \hat{g}_{3}\right)+\left(\beta^{2}+1\right)\left(\hat{g}_{2}^{2}+\hat{g}_{3}^{2}\right)}{\beta^{2}\left(\hat{g}_{4}^{2}-1\right)}
\end{array}\right) .
\end{aligned}
$$


A very complicated subsequent transformation brings the metric to Brinkmann form

$$
d s^{2}=-\left(\frac{1+2 \beta^{2} \operatorname{sech}^{2}(u)}{\beta^{2}}\right)\left(z_{3}^{2}+z_{4}^{2}\right) d u^{2}+2 d u d v+d z_{3}^{2}+d z_{4}^{2}
$$

for $\left|\hat{g}_{4}\right|<1$, or

$$
d s^{2}=-\left(\frac{1-2 \beta^{2} \operatorname{csch}^{2}(u)}{\beta^{2}}\right)\left(z_{3}^{2}+z_{4}^{2}\right) d u^{2}+2 d u d v+d z_{3}^{2}+d z_{4}^{2}
$$

for $\left|\hat{g}_{4}\right|>1$. The corresponding torsion of this model is

$$
\widehat{\mathcal{H}}=\frac{2}{\beta} d u \wedge d z_{3} \wedge d z_{4}
$$

This restores the results of [15].

\section{Dualization with respect to subgroup generated by $S_{4,29}$}

We choose $\xi(s)=\left(-\frac{1}{2}, 0,0,-\frac{1}{2}\right)$. When conditions (60) hold, i.e. when $B_{4}=B_{5}=0$, the shift

$$
\hat{g}_{2} \mapsto \hat{g}_{2}+B_{1} \cot (\gamma), \quad \hat{g}_{3} \mapsto \hat{g}_{3}-B_{3}, \quad \hat{g}_{4} \mapsto \hat{g}_{4}+B_{2}
$$

simplifies the dual background to the form

$$
\widehat{\mathcal{F}}\left(\hat{g}_{1}, \hat{g}_{2}, \hat{g}_{3}, \hat{g}_{4}\right)=\left(\begin{array}{cccc}
0 & \frac{\cot (\gamma)}{\hat{g}_{2}+1} & 0 & 0 \\
\frac{\cot (\gamma)}{1-\hat{g}_{2}} & \frac{\cot ^{2}(\gamma)\left(\hat{g}_{3}^{2}+\hat{g}_{4}^{2}\right)}{\left(B_{6}^{2}+1\right)\left(\hat{g}_{2}^{2}-1\right)} & \frac{\cot (\gamma)\left(B_{6} \hat{g}_{3}-\hat{g}_{4}\right)}{\left(B_{6}^{2}+1\right)\left(\hat{g}_{2}-1\right)} & \frac{\cot (\gamma)\left(\hat{g}_{3}+B_{6} \hat{g}_{4}\right)}{\left(B_{6}^{2}+1\right)\left(\hat{g}_{2}-1\right)} \\
0 & -\frac{\cot (\gamma)\left(B_{6} \hat{g}_{3}+\hat{g}_{4}\right)}{\left(B_{6}^{2}+1\right)\left(\hat{g}_{2}+1\right)} & \frac{1}{B_{6}^{2}+1} & -\frac{B_{6}}{B_{6}^{2}+1} \\
0 & \frac{\cot (\gamma)\left(\hat{g}_{3}-B_{6} \hat{g}_{4}\right)}{\left(B_{6}^{2}+1\right)\left(\hat{g}_{2}+1\right)} & \frac{B_{6}}{B_{6}^{2}+1} & \frac{1}{B_{6}^{2}+1}
\end{array}\right)
$$

where $0<\gamma<\pi, \gamma \neq \frac{\pi}{2}$. For $\left|\hat{g}_{2}\right|<1$ subsequent coordinate transformation

$$
\begin{array}{ll}
\hat{g}_{1}=v+\frac{1}{2}\left(B_{6}\left(z_{3}^{2}+z_{4}^{2}\right)\right), & \hat{g}_{3}=\sqrt{B_{6}^{2}+1}\left(z_{3} \cos \left(f_{\gamma}(u)\right)-z_{4} \sin \left(f_{\gamma}(u)\right),\right. \\
\hat{g}_{2}=\tanh (u \tan (\gamma)), & \hat{g}_{4}=\sqrt{B_{6}^{2}+1}\left(z_{4} \cos \left(f_{\gamma}(u)\right)+z_{3} \sin \left(f_{\gamma}(u)\right)\right)
\end{array}
$$

where

$$
f_{\gamma}(u)=\cot (\gamma) \log (\cosh (u \tan (\gamma)))
$$

brings this pp-wave metric to Brinkmann form (40). The corresponding torsion of this model is (41). For $\left|\hat{g}_{2}\right|>1$ similar transformation with tanh $\rightarrow$ coth and cosh $\rightarrow$ sinh again gives metric (40) and torsion (41).

Dualization with respect to subgroups generated by $S_{4,31}, S_{4,33}$

As we can see from Tab. 4. subalgebras $S_{4,31}$ and $S_{4,33}$ contain the parameter $\beta \neq 0$ and differ only in the value of the parameter $\alpha$. Accordingly, the results of dualization and corresponding coordinate transformations can be written in terms of $\alpha$, which is positive 
for $S_{4,33}$ and vanishes for $S_{4,31}$. We choose $\xi(s)=\left(-\frac{1}{2}, 0,0,-\frac{1}{2}\right)$. For both subalgebras the conditions (60) hold when $B_{4}=B_{6}=0$ and $B_{5}=B_{2}$. The scalar curvatures $\widehat{\mathcal{R}}$ then vanish. After the shift

$$
\hat{g}_{3} \mapsto \hat{g}_{3}-B_{2}, \quad \hat{g}_{4} \mapsto \hat{g}_{4}-B_{3}
$$

the dual background gets the form

$$
\begin{aligned}
& \widehat{\mathcal{F}}\left(\hat{g}_{1}, \hat{g}_{2}, \hat{g}_{3}, \hat{g}_{4}\right)= \\
& \left(\begin{array}{cccc}
0 & 0 & -\frac{1}{\hat{g}_{3}+1} & 0 \\
0 & \frac{1}{\beta^{2}+\hat{g}_{3}^{2}} & \frac{\left.\alpha \beta-\hat{g}_{3}+1\right) \hat{g}_{4}}{\left(\hat{g}_{3}+1\right)\left(\beta^{2}+\hat{g}_{3}^{2}\right)} & -\frac{\hat{g}_{3}+1}{\beta^{2}+\hat{g}_{3}^{2}} \\
\frac{1}{\hat{g}_{3}-1} & -\frac{\alpha \beta+B_{1}+\left(\hat{g}_{3}-1\right) \hat{g}_{4}}{\left(\hat{g}_{3}-1\right)\left(\beta^{2}+\hat{g}_{3}^{2}\right)} & \frac{B_{1}^{2}-2 \hat{g}_{4} B_{1}+\alpha^{2} \hat{g}_{3}^{2}+\left(\beta^{2}+1\right) \hat{g}_{4}^{2}-2 \alpha \beta \hat{g}_{3} \hat{g}_{4}}{\left(\hat{g}_{3}^{2}-1\right)\left(\beta^{2}+\hat{g}_{3}^{2}\right)} & \frac{\alpha \beta\left(\hat{g}_{3}+1\right)+B_{1}\left(\hat{g}_{3}+1\right)-\left(\beta^{2}+1\right) \hat{g}_{4}}{\left(\hat{g}_{3}-1\right)\left(\beta^{2}+\hat{g}_{3}^{2}\right)} \\
0 & \frac{\hat{g}_{3}-1}{\beta^{2}+\hat{g}_{3}^{2}} & \frac{\alpha \beta\left(\hat{g}_{3}-1\right)-B_{1}\left(\hat{g}_{3}-1\right)-\left(\beta^{2}+1\right) \hat{g}_{4}}{\left(\hat{g}_{3}+1\right)\left(\beta^{2}+\hat{g}_{3}^{2}\right)} & \frac{\beta^{2}+1}{\beta^{2}+\hat{g}_{3}^{2}}
\end{array}\right) .
\end{aligned}
$$

A very complicated subsequent transformation brings the metric to the following $\alpha$ independent Brinkmann form

$$
\begin{gathered}
d s^{2}=\left(\frac{4\left(-2 \beta^{2}+\left(\beta^{2}+1\right) \cosh (2 u)-1\right)}{\left(\beta^{2}+\left(\beta^{2}+1\right) \cosh (2 u)-1\right)^{2}} z_{3}^{2}-\frac{4 \beta^{2}\left(\beta^{2}+\left(\beta^{2}+1\right) \cosh (2 u)+2\right)}{\left(\beta^{2}+\left(\beta^{2}+1\right) \cosh (2 u)-1\right)^{2}} z_{4}^{2}\right) d u^{2} \\
+2 d u d v+d z_{3}^{2}+d z_{4}^{2}
\end{gathered}
$$

for $\left|\hat{g}_{4}\right|<1$, with corresponding torsion

$$
\widehat{\mathcal{H}}=\frac{4 \beta}{\beta^{2}+\left(\beta^{2}+1\right) \cosh (2 u)-1} d u \wedge d z_{3} \wedge d z_{4},
$$

or to the form

$$
\begin{gathered}
d s^{2}=\left(-\frac{4\left(2 \beta^{2}+\left(\beta^{2}+1\right) \cosh (2 u)+1\right)}{\left(-\beta^{2}+\left(\beta^{2}+1\right) \cosh (2 u)+1\right)^{2}} z_{3}^{2}+\frac{4 \beta^{2}\left(-\beta^{2}+\left(\beta^{2}+1\right) \cosh (2 u)-2\right)}{\left(-\beta^{2}+\left(\beta^{2}+1\right) \cosh (2 u)+1\right)^{2}} z_{4}^{2}\right) d u^{2} \\
+2 d u d v+d z_{3}^{2}+d z_{4}^{2}
\end{gathered}
$$

for $\left|\hat{g}_{4}\right|>1$, with corresponding torsion

$$
\widehat{\mathcal{H}}=\frac{4 \beta}{-\beta^{2}+\left(\beta^{2}+1\right) \cosh (2 u)+1} d u \wedge d z_{3} \wedge d z_{4} .
$$

Although it is not obvious at first sight, this restores the results of [15].

\section{References}

[1] T. H. Buscher, A symmetry of the String Background Field Equations, Phys. Lett. B 194 (1987) 51.

[2] X. C. de la Ossa and F. Quevedo, Duality symmetries from non-abelian isometries in string theories, Nucl. Phys. B 403 (1993) 377, hep-th/9210021. 
[3] C. Klimčík and P. Ševera, Dual non-Abelian duality and the Drinfeld double, Phys. Lett. B 351 (1995) 455, hep-th/9502122.

[4] G. Cavalcanti and M. Gualtieri, Generalized complex geometry and T-duality, In: A Celebration of the Mathematical Legacy of Raoul Bott (CRM Proceedings \& Lecture Notes), American Mathematical Society (2010) 341, arXiv:1106.1747.

[5] P. Ševera, Poisson-Lie T-Duality and Courant Algebroids, Lett. in Mat. Phys. 105 (12) (2015) 1689, arXiv:1502.04517.

[6] P. Bouwknegt, M. Bugden, C. Klimčík, and K. Wright, Hidden isometry of "Tduality without isometry", JHEP 2017 (8) (2017) 116, arXiv:1705.09254.

[7] S. F. Hassan, T-Duality, Space-time Spinors and R-R Fields in Curved Backgrounds, Nucl. Phys. B 568 (2000) 145, hep-th/9907152.

[8] K. Sfetsos and D. C. Thompson, On non-abelian T-dual geometries with Ramond fluxes, Nucl. Phys. B846 (2011) 21, arXiv:1012.1320.

[9] Y. Lozano, E. O'Colgain, K. Sfetsos, and D. C. Thompson, Non-abelian T-duality, Ramond fields and coset geometries, JHEP 1106 (2011) 106, arXiv:1104.5196.

[10] G. Itsios, C. Nunez, K. Sfetsos, and D. C. Thompson, Non-Abelian T-duality and the AdS/CFT correspondence:new N=1 backgrounds, Nucl. Phys. B 873, 1 (2013), arXiv:1301.6755.

[11] D. Sadri, M.M. Sheikh-Jabbari, The Plane-Wave/Super Yang-Mills Duality, Rev.Mod.Phys. 76 (2004) 853, [hep-th/0310119].

[12] R. Borsato, L. Wulff, Integrable deformations of T-dual $\sigma$-models, Phys. Rev. Lett. 117, 251602 (2016), arXiv:1609.09834.

[13] R. Borsato, L. Wulff, On non-abelian T-duality and deformations of supercoset string sigma-models, JHEP 2017 (24) (2017), arXiv:1706.10169.

[14] F. Petrásek, L. Hlavatý, and I. Petr, Plane-parallel waves as duals of the flat background II: T-duality with spectators, Class. Quantum Grav. 34 (2017) 155003, arXiv:1612.08015.

[15] L. Hlavatý and I. Petr, Plane-parallel waves as duals of the flat background, Class. Quantum Grav. 32 (2015) 035005, arXiv:1406.0971.

[16] J. Patera, R. T. Sharp, P. Winternitz, and H. Zassenhauss, Subgroups of the Poincaré group and their invariants, J. Math. Phys. 17 (1976) 977.

[17] E. Alvarez, L. Alvarez-Gaume, J. L. F. Barbon, and Y. Lozano, Some Global Aspects of Duality is String Theory, Nucl.Phys. B415 (1994) 71, hep-th/9309039|.

[18] A. A. Tseytlin, Exact solutions of closed string theory, Class. Quant. Grav. 12 (1995) 2365, hep-th/9505052]. 
[19] A. A. Tseytlin, Exact string solutions and duality, Proc. Cosmology, Paris (1994) 371, hep-th/9407099.

[20] K. Sfetsos and A. A. Tseytlin, Four Dimensional Plane Wave String Solutions with Coset CFT Description, Nucl. Phys. B 427 (1994) 245, hep-th/9404063.

[21] M. Blau, M. O’Loughlin, G. Papadopoulos, and A. A. Tseytlin, Solvable models of strings in homogeneous plane wave backgrounds, Nucl. Phys. B 673 (2003) 57, hep-th/0304198.

[22] G. Papadopoulos, J. G. Russo, and A. A. Tseytlin, Solvable model of strings in a time-dependent plane-wave background, Class. Quantum Grav. 20 (2003) 969, hep-th/0211289.

[23] G. Itsios, Y. Lozano, E. O'Colgain, and K. Sfetsos, Non-Abelian T-duality and consistent truncations in type-II supergravity, JHEP 1208 (2012) 132, [arXiv:1205.2274].

[24] H. Dimov, S. Mladenov, R. C. Rashkov, and T. Vetsov Non-abelian T-duality of Pilch-Warner background, Fortschr. Phys. 64, 657 (2016), arXiv:1511.00269.

[25] Y. Lozano, C. Nunez, and S. Zacarias, BMN Vacua, Superstars and Non-Abelian T-duality, JHEP 2017 (0) (2017), arXiv:1703.00417].

[26] L. Hlavatý and F. Petrásek, On uniqueness of T-duality with spectators, Int. J. Mod. Phys. A 31 (2016) 1650143, arXiv:1606.02522.

[27] M. Blau, J. Figueroa-O'Farrill, and G. Papadopoulos, Penrose limits, supergravity and brane dynamics, Class. Quantum Grav. 19 (2002) 4753, hep-th/0202111.

[28] R. Penrose, Any Space-Time has a Plane Wave as a Limit, in: Differential Geometry and Relativity, vol. 3, eds. M. Cahen and M. Flato, Reidel, Dordrecht 1976.

[29] R. Gueven, Plane wave limits and T-duality, Phys. Lett. B 482 (2000), 255 - 263, hep-th/0005061v1].

[30] I. Antoniadis and N.A. Obers, Plane Gravitational Waves in String Theory, Nucl. Phys. B423 (1994) 639, hep-th/9403191. 